\title{
DEVELOPMENT AND VALIDATION OF METHODS \\ FOR SAMPLING AND ANALYSIS OF \\ WORKPLACE TOXIC SUBSTANCES
}

\author{
E11en C. Gunderson \\ C. Clarine Anderson \\ SRI International \\ Menlo Park, California 94025 \\ with contributions by \\ Richard H. Smith (Arthur D. Little, Inc.) \\ Laurence J. Doemeny (NIOSH)
}

Contract No. 210-76-0123

U.S. DEPARTMENT OF HEALTH AND HUMAN SERVICES

Public Health Services

Center for Disease Control

National Institute for Occupational Safety and Health

Division of Physical Sciences and Engineering

Cincinnati, Ohio 45226

September 1980 
The contents of this report are reproduced herein as received from the contractor.

The opinions, findings and conclusions expressed herein are not necessarily those of the National Institute for Occupational Safety and Health, nor does mention of company names or products constitute endorsement by the National Institute for Occupational Safety and Health.

NIOSH Project Officer: Laurence J. Doemeny Alternate Project Officer: David G. Taylor

Project Director: Dale M. Coulson

SRI International Task Leaders:

E11en C. Gunderson

C. Clarine Anderson

Arthur D. Little, Inc. Task Leaders:

Philip L. Levins

Richard H. Smith

Alegria B. Caragay

DHHS (NIOSH) Publication No. 80-133 


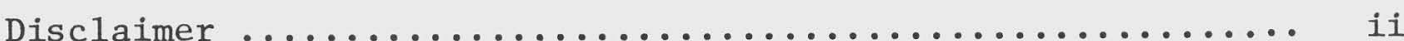

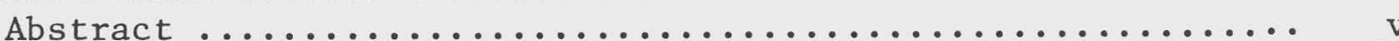

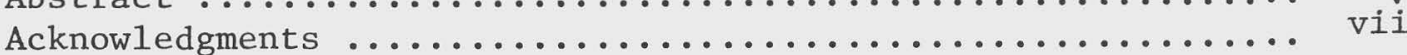

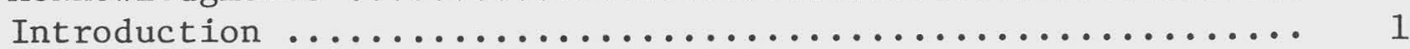

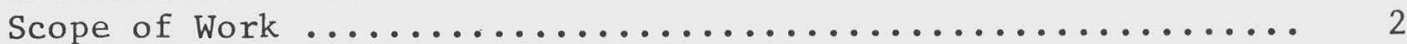

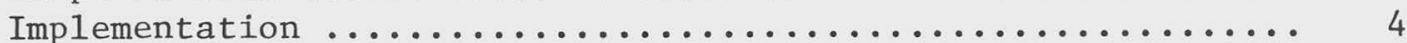

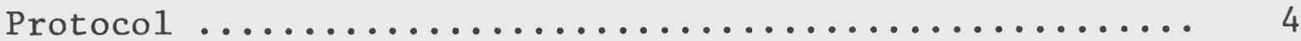

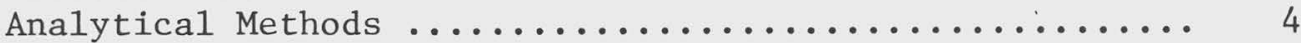

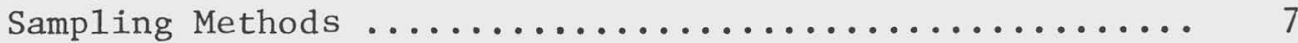

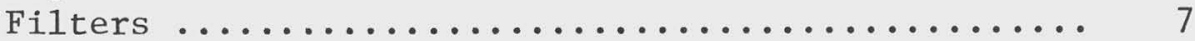

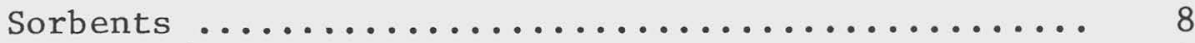

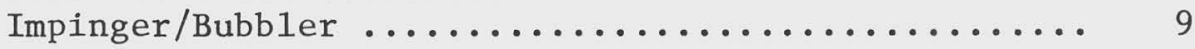

Particulate/Vapor Sampling Methods ............. 9

Other Sampling Methods ................... 10

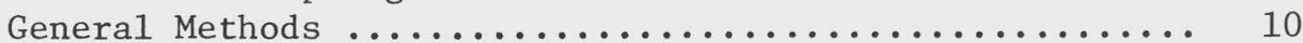

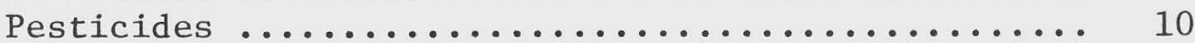

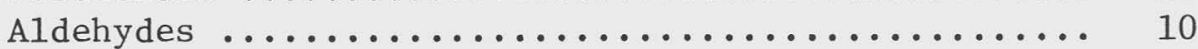

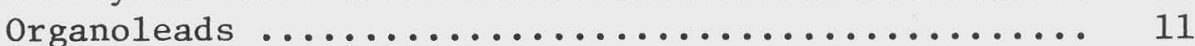

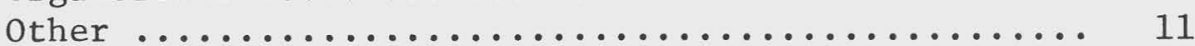

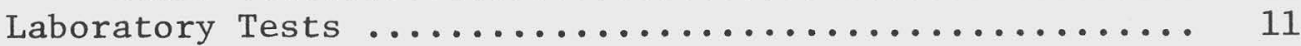

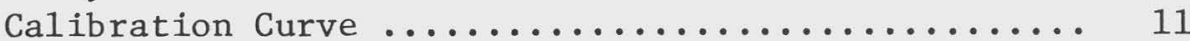

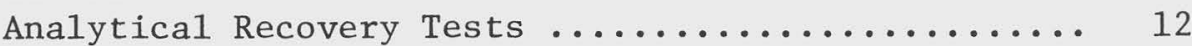

Determination of Breakthrough Volume or

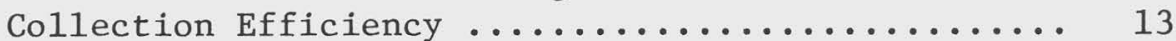

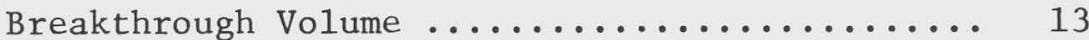

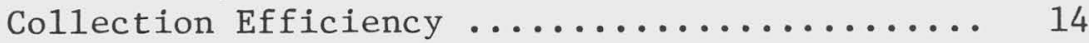

Generation and Collection of Samples ................ 14

Independent Method of Determining Test Air

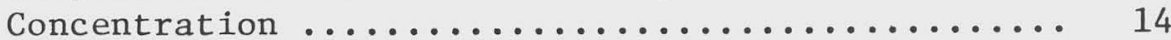

Storage Stability of Generated Samples .......... 15

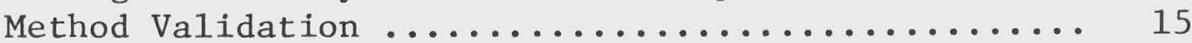

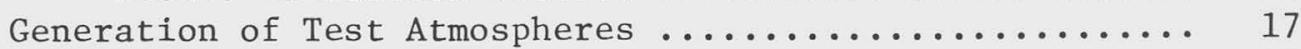

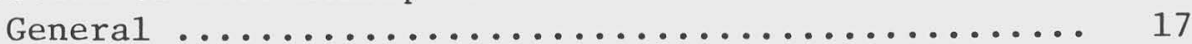

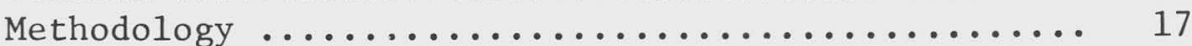

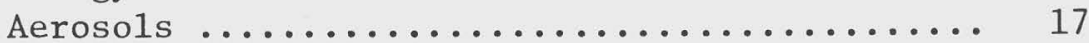

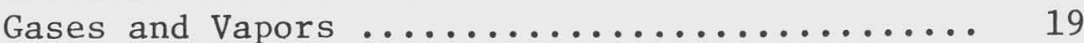

Main Dilution/Sampling System .............. 20

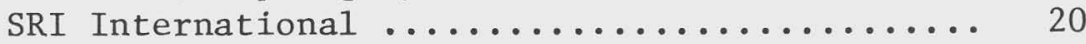

Arthur D. Little, Inc. Gas and Vapor System ... 24

Arthur D. Little, Inc. Aerosol System ....... 26

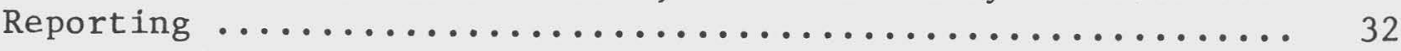

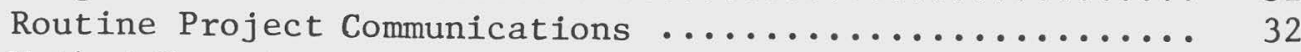

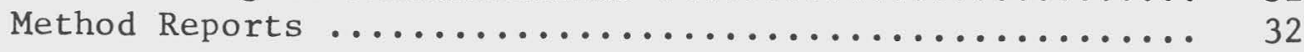


Delivery and Review of Draft Reports ............. 33

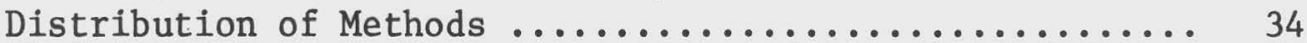

Conclusions and Recommendations .................. 35

Appendix A ................................... A-1

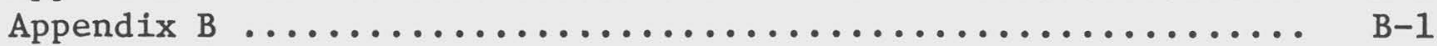




\section{ABSTRACT}

To determine the level of exposure of workers to toxic substances, adequate sampling and analytical methods are required for the specific substances. The objective of this study was to develop and validate methods for sampling and analyzing 130 substances for which no methods were currently available. This report summarizes the experimental protocol and methodology used in the method development and validation effort.

This report was submitted in fulfillment of Contract No. 210-76-0123 by SRI International and its subcontractor, Arthur D. Little, Inc., under the sponsorship of the National Institute for Occupational Safety and Health. 


\section{ACKNOWLEDGMENTS}

The authors would like to acknowledge the staff members at SRI International, Arthur D. Little, Inc., and the National Institute for Occupational Safety and Health for their efforts in contributing toward the overall success of this project. At SRI we especially recognize and greatly appreciate the dedicated efforts of Dr. Dale M. Coulson, Ellen L. Fernandez, and Jolene Y. Louie, in addition to the following people:

Jennifer E. Barnett
Curtis W. Beeman
Ronald W. Buckley
S. Sam Fratoni
Lois L. Gerchman
Mary J. Grubic
Daniel L. Haynes
Erick L. Hagmann

Lawrence B. Inman

Charles E. Lapple

Ilsabe C. Niemeyer

James H. Smith

Douglas S. Stivers

Willoughby Thorn

E1 Lorraine Watson

At ADL, we gratefully acknowledge the work performed by Richard H. Smith, Dr. Philip L. Levins, Alegria B. Caragay, and Christine M. Freitas, in addition to:

\author{
Itamar Bodek \\ Laurence T. Damokosh \\ Martha G. Fernald \\ Bruce E. Goodwin \\ Joan B. Hastings
}

\author{
Kenneth T. Menzies \\ Virginia A. Sherman \\ Joel Weddig \\ Ruth H. Willson \\ Doris Wilson
}

Throughout the research effort we have appreciated very much the guidance and support provided by the Project Officer, Dr. Laurence J. Doemeny. His efforts have helped to make this program a very interesting and rewarding experience. Also at NIOSH we especially recognize the continued efforts and suggestions provided by Dr. David G. Taylor and Mr. William A. Heitbrink and the method reviews provided by:

Martin A. Abel1 Mark F. Boeniger Michele Bolyard Thomas P. Carsey

Mary E. Cassinelli

Clinton Cox

Harry M. Donaldson

Peter M. Eller

Denis L. Foerst

Robert A. Glaser

Janet C. Haartz

Robert H. Hill, Jr.

R. Delon Hul1
James H. Jones

Bruce Lange

Howard R. Ludwig

Alan A. Lunsford

Charles S. McCammon, Jr.

James L. Oser

Judd C. Posner

Martha J. Seymour

Doris V. Sweet

Alexander $W$. Teass

Samue1 P. Tucker

Allen W. Verstuyft 
We finally extend our thanks to Grace A. Fannin who kept many of the NIOSH staff appraised of the status of the methods and distributed the methods for review and dissemination. 


\section{INTRODUCTION}

The Occupational Safety and Health Act of 1970 (Public Law 91-596) requires monitoring and the maintaining of records to determine if and when employees are exposed to toxic hazards in the workplace. Exposure standards have been established for certain substances by the Occupational Safety and Health Administration (OSHA). The National Institute for Occupational Safety and Health (NIOSH) is responsible under the Act for providing reliable sampling and analytical procedures in order that the work environment can be monitored to determine compliance.

For many years the level of employee exposure to toxic substances has been determined by collecting an air sample within the breathing zone of the worker. Because most of the air standards are 8-hour time-weighted averages (TWA) and because workers move about, personal, portable sampling and collection systems have been required. Since some air standards are ceiling and/or peak values, they require additional samples collected over short time intervals ( 15 minutes) to determine if an exposure might occur at or above the maximum allowable levels.

To ensure the established standards, valid sampling and analytical methods must be available. In 1974, as part of a joint effort by NIOSH and OSHA to complete the existing Standards (29CRF 1910.1000), NIOSH entered into a contract to validate sampling and analytical methods for 386 compounds (NIOSH Contract No. CDC99-47-45). This total effort, known as the Standards Completion Program, was completed in September 1976. In the method validation part of the total effort, methods were not validated for 170 substances because of nonavailability of methods, contractual constraints, statistical requirements, or other reasons.

For some substances, methods may never be developed; for others, storage, recovery, precision or accuracy requirements make it unlikely that validation would be achieved. Therefore, 130 compounds were selected for additional work. The experimental protocol and methodology described herein formed the basis for the development and validation of sampling and analytical methods for these 130 substances.

Substances for which NIOSH had planned to prepare criteria documents were given highest priority and work was scheduled early in the contract. Other substances were scheduled to coordinate with the completion of NIOSH in-house and contract work. 


\section{SCOPE OF WORK}

The protocol for validation of each sampling and analytical method consisted of a two-part experimental study. An analytical method was first developed and tested for the substance. The sensitivity and specificity of the method were evaluated and the response to known interferences was determined where necessary. After this, an appropriate sample collection medium was chosen. In the case of particulates, a suitable filter was determined; for gases or vapors, an absorption liquid or, preferably, solid sorbent was chosen. For particulate/vapor mixtures, multiple stage sampling trains were developed. The sampling medium was spiked with known amounts of the test substance at three levels corresponding to one-half, one, and two times the OSHA environmental limit at a given air volume. An efficient recovery procedure, such as solvent desorption/extraction, was developed. The analytical recovery of the substance from the sampling medium was then determined in the spiking experiments. In addition, the precision of the analytical method was evaluated.

If the analytical method were acceptable in recovery and precision, the second part of the validation effort was initiated. This part involved testing the efficiency of the sampling procedure with known air concentrations of the toxic substance prepared in a laboratory generation-dilution system. For sorbents, the breakthrough volume was also evaluated. After the optimum sampling flow rate and sample volume were determined, samples were collected from a laboratory generation-dilution system. The concentration of the generated test atmosphere was determined by an independent method of measurement. The stability of collected samples was evaluated by taking the results obtained from samples that had been analyzed immediately after collection and comparing them to samples that had been analyzed after a storage period. Based on the results of the validation tests, an evaluation was made of the precision and accuracy of the analytical method itself and of the sampling and analytical method as a whole.

The basic criterion for successful validation was that a method should come within $\pm 25 \%$ of the "true value" at the $95 \%$ confidence level. To meet this criterion, the protocol for experimental testing and method validation was established with a firm statistical basis. A statistical protocol provided methods of data analysis that allowed the accuracy criterion to be evaluated with statistical parameters estimated from the laboratory test data. It also gave a means to evaluate precision and bias, independently and in combination, to determine the accuracy of sampling and analytical methods.

The substances studied in this program are summarized in Table 1. 


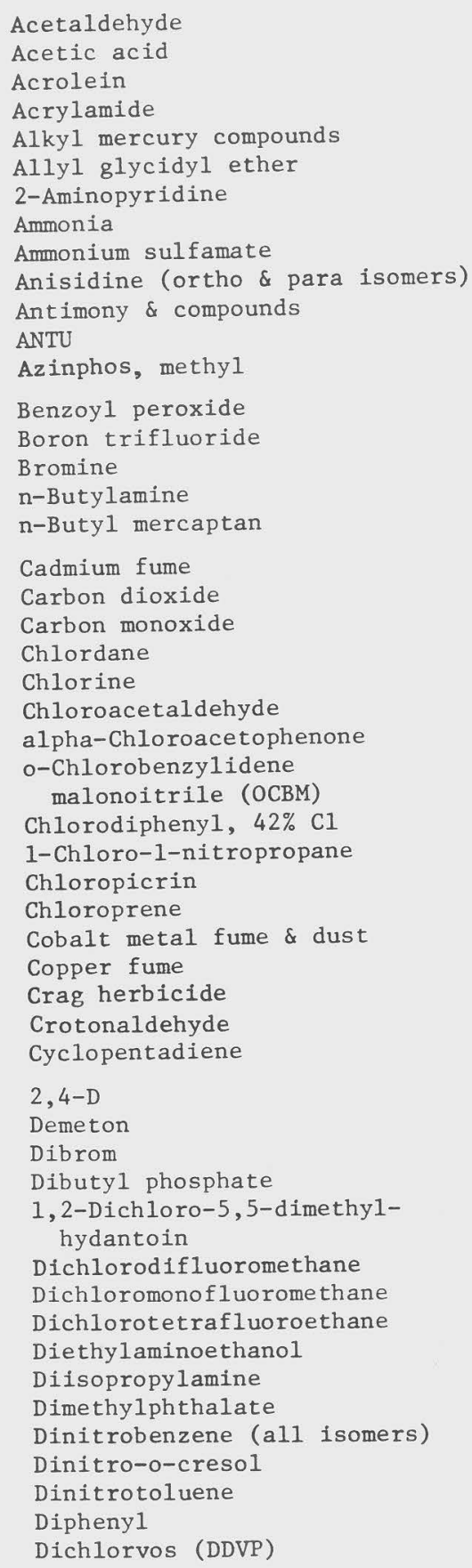

Endrin

Ethanolamine

2-Ethoxyethanol

Ethyl chloride

Ethylenediamine

Ethyl mercaptan

Ferbam

Fluorides in dust

Fluorine

Fluorotrichloromethane

Formaldehyde

Formic acid

Furfural

Furfuryl alcohol

Hafnium

Heptachlor

Hydrogen cyanide

Hydroquinone

Iodine

Iron oxide fume

Malathion

Maleic anhydride

MAPP (methyl acetylene/ propadiene mix)

Mercury

Methoxychlor

Methyl acetylene

Methylamine

Methyl chloride

Methylcyclohexanol

Methylcyclohexanone

Methyl formate

Methyl isocyanate

Methy1 mercaptan

Methyl methacrylate

Nitric acid

Nitric oxide

$\mathrm{p}$-Nitroaniline

Nitroethane

Nitromethane

Nitrogen dioxide

I-Nitropropane

Oxalic acid

Paraquat

Pentachlorophenol

Perchloromethyl mercaptan

p-Phenylenediamine

Phosdrin

Phosphine

Phosphorus pentachloride
Phosphorus yellow

Pyrethrum

Picric acid

Pival

Quinone

Ronne1

Rotenone

Silver, metal and soluble compounds

Sodium fluoroacetate

Sodium hydroxide

Stibine

Strychnine

Sulfur dioxide

Sulfur hexafluoride

Sulfur monochloride

Sulfuryl fluoride

$2,4,5-T$

TEDP

TEPP

Tantalum

Tetraethyl lead

Tetramethy 1 lead

Thiram

Tin, organic compounds

2,4-Toluenediisocyanate

Trinitrotoluene

Vanadium, $\mathrm{V}_{2} \mathrm{O}_{5}$ dust

Vanadium fume

Warfarin

Zinc oxide fume 


\section{IMPLEMENTATION}

\section{PROTOCOL}

If no specific method was designated by NIOSH for a substance, the study began with a review of the failure report and previous experimental work performed on the substance under NIOSH Contract No. CDC-99-74-45. If necessary, a 1iterature search was performed covering the following essentials:

- Physical and chemical properties.

- Sampling methods, including preservation of collected samples, and derivatization procedures where applicable.

- Analytical methods, including specific techniques for separation, detection, and quantification by gas or liquid chromatography, atomic absorption, or other methods.

An experimental protocol for method development was prepared before starting laboratory work. The general procedure, involving several interdependent experiments, is illustrated in Figure 1. The protocol allowed for efficient decisionmaking on a step-by-step basis. Since adverse results in any one step could cause any previous experimentation to be rejected, it was extremely important in any protocol to eliminate potential problems at the earliest stage possible. A specific plan was therefore designed so that the most vulnerable point of the proposed sampling and analytical method would be tested early in the procedure. For example, if storage stability of collected samples or capacity of the sampling media were suspected problems, then these factors were investigated in early experiments. When a method failed to meet accuracy or precision criteria at one stage of the validation procedure, work then reverted to an earlier stage. How far work regressed was determined by the nature of the problem, the point in the protocol at which the experiment failed, and the time available for additional work. The level of effort for method development and validation was such that no more than an average of 60 professional man-days with a maximum of 75 professional man-days were to be expended on each compound. At various decisionmaking points in the protocol, meetings were held with project management to discuss the status of work and plan for immediate testing. Consultation between NIOSH, SRI, and ADL was also valuable in solving problems in the refinement of a method.

\section{ANALYTICAL METHODS}

The criteria for choosing an analytical method in this program included the use of modern instrumentation and chemical methods that were specific for the substance analyzed. The method should also lend itself to routine analysis of a large number of samples. Where possible, the method should be economical, and 


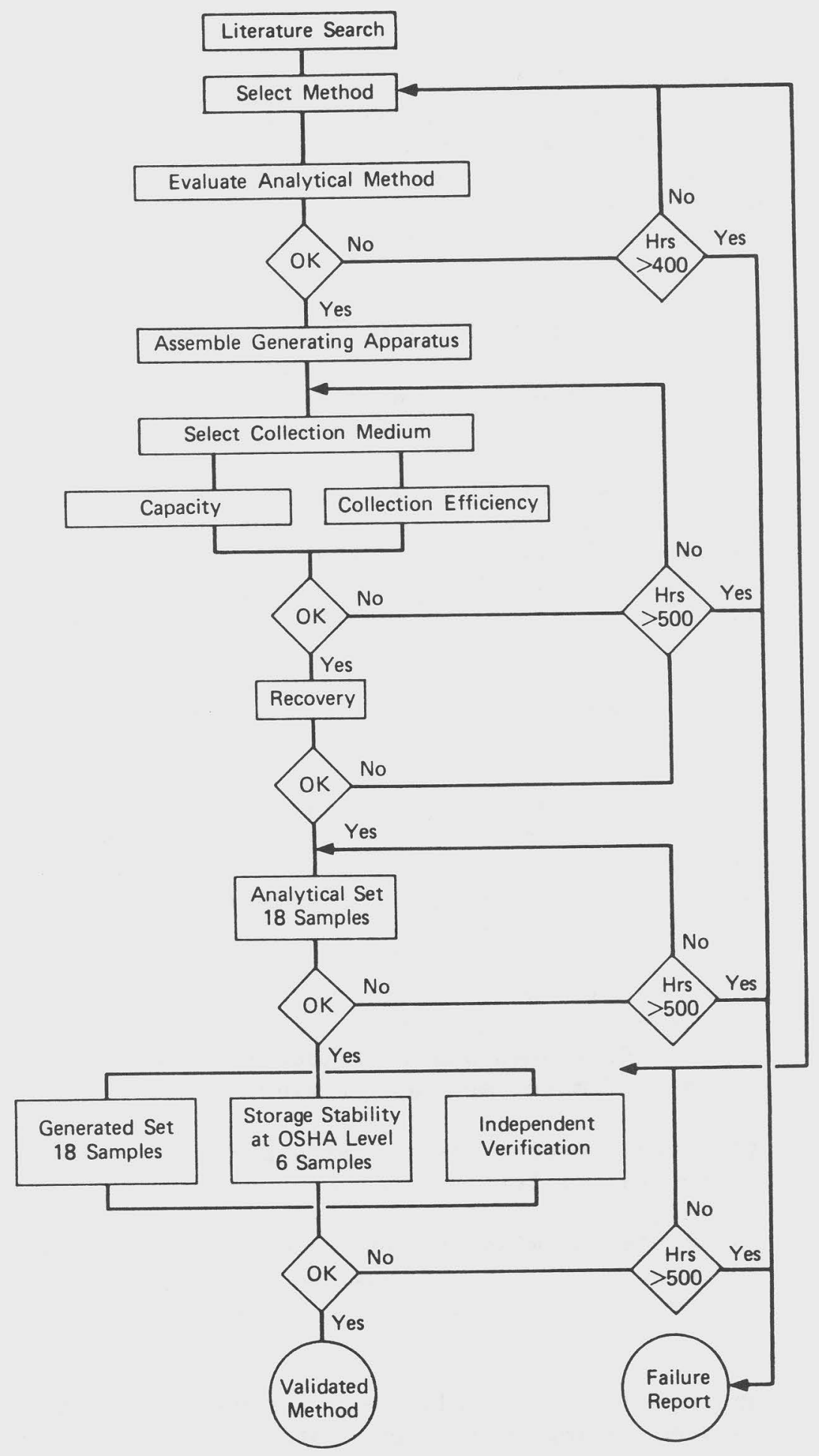

FIGURE 1 PROTOCOL FOR METHOD DEVELOPMENT AND VALIDATION 
easily applied by experienced technicians with technical school or some college experience. When such methods were not available, more sophisticated methods were investigated.

Analytical methods were chosen on the basis of selectivity and sensitivity. The method chosen influenced the choice of the sampling system and the solvents used in the analysis. Solvents and sampling media were selected for compatibility with the specific detector or analytical system rather than vice versa. In general, this policy allowed lower levels of detection. In selecting analytical methods, the effects of known interferences were also considered. For example, in gas chromatography, columns were chosen to optimize resolution from these interferences.

The majority of analytical methods developed in the validation studies utilized gas chromatography (GC) and high-performance liquid chromatography (HPLC). Gas chromatographic analysis was the predominent analytical method of choice for volatile organic compounds. Since many of the substances investigated were volatile organic compounds, GC coupled with selective detectors provided very sensitive and selective analytical methods. Detectors used in this program included thermal conductivity, flame ionization, electron capture, electrolytic conductivity, flame photometric, alkali flame ionization, and photoionization. Commonly used GC packings were used. Glass GC columns were necessary for many substances. In most cases, samples of the analyte in a solvent were injected into the GC by syringe, either by hand or automatic injector. A gas sampling loop was used to inject samples collected in bags. A specially designed apparatus was fabricated to introduce samples into the GC from a sorbent tube by thermal desorption.

HPLC with UV absorption detection was frequently used in this program. This method was suitable for less volatile organic substances that were thermally unstable, had high UV absorptivity, or were particularly suited to liquid chromatography. Separation modes used included absorption (normal phase), partition in normal and reverse phases, ion exchange, and paired-ion chromatography.

Ion chromatography (IC) coupled with conductimetric detection provided an efficient and sensitivie method of analyzing specific organic or inorganic ionic species. IC methods were developed and validated for formic acid, sodium fluoroacetate, ammonium sulfamate, and methylamine.

Atomic absorption (AA) spectroscopy was the method of choice for most metals and metallic compounds. Several different excitation sources were used in addition to standard flame techniques, including the electrodeless discharge lamp and graphite furnace. A dc plasma emission spectrometer was used in the analysis of hafnium, tantalum and silver. For these compounds, the concentration levels of interest were too low for conventional AA analysis to be of sufficient precision and accuracy.

Colorimetric methods were developed only when reproducibility and selectivity of the method represented an improvement over other methods. This was the case for stibine and phosphorous pentachloride. In the case of phosphine, a stable derivative was formed that was particularly suited to colorimetry. 
Ion-selective electordes were used in the program. Methods were validated based on this technique for ammonia, hydrogen cyanide, and nitric acid. In addition, titrations were used for sodium hydroxide and sulfur dioxide.

Other methods were based upon particular characteristics of the analyte. For example, x-ray diffraction was applied to the analysis of zinc oxide.

\section{SAMPLING METHODS}

Industrial hygiene sampling and analysis are used to determine the concentration of chemicals to which employees are exposed. Collection of a representative sample is difficult and is best done using personal sampling devices that collect samples from the employee's breathing zone. Such devices normally consist of a sample collection device (filter or sorbent tube) worn at the lapel and connected by flexible tubing to a calibrated sampling pump worn on the belt. Personal sampling pumps that operate in several ranges from 0.01 to $4 \mathrm{~L} / \mathrm{min}$ are available. These pumps can collect both short- and long-term samples for evaluation of exposure to ceiling levels and the time-weighted average.

The first consideration is the selection of a sampling medium compatible with the physical state of the analyte at the levels of interest. The substance may exist as a gas, vapor, particulate, or combination, depending on the level in a given atmosphere. At low levels, one compound may exist as a vapor, but at higher levels it may be primarily particulate. Predictions of vapor concentrations may be derived from vapor pressure data, if available, but for some substances no reliable data are available.

A rough guideline was used in this program for determining if a substance exists as a vapor or particulate (or both) at levels near the OSHA standard. If the ratio of the equilibrium vapor concentration at $25^{\circ} \mathrm{C}$ to the standard were between 0.05 and 50, then contribution from both vapor and particulate was considered. A ratio below 0.05 indicates that the material will probably be particulate; whereas, if above 50, it will probably be vapor. Frequently, this determination was made experimentally by generating test atmospheres of the substance near the standard and collecting samples with particulate/vapor sampling trains.

The sampling devices most commonly used were filters, solid sorbent tubes (charcoal, silica gel, porous polymers), and midget bubblers and impingers. In terms of ease and reliability, the first two methods were considered most desirable. Filters collect particulates and sorbent tubes collect gases and vapors, a1though there are also indications that some vapors will collect on filters, and glass wool plugs used to retain sections of sorbent may collect a portion of the particulates. Some reactive species had to be stabilized at the time of sampling, frequently by trapping them in a suitable solvent in impingers or bubblers because no reliable alternative procedures were available. These devices are troublesome to use in the field and were avoided when possible.

\section{Filters}

The procedure most commonly used for collection of particulates involves sample collection with a closed-face $37-\mathrm{mm}$ filter cassette containing a filter supported by a cellulose backup filter support. The most frequently used filter materials include mixed cellulose ester (MCE), polyvinyl chloride (PVC), glass 
fiber, and recently, polytetrafluoroethylene (PTFE). Any high-efficiency filter usually functions well for collection purposes; therefore, selection was usually based on the final analytical requirements. However, in this program, poor recoveries were observed for some substances (such as thiram) when glass fiber filters were used, and the filter medium was found to be at fault. The PTFE filters, being inert, were very useful for collecting reactive substances. In the analytical method for sodium fluoroacetate by ion chromatography, surfactants used as wetting agents on some filters were found to significantly interfere.

MCE filters were generally used when the sample was extracted, digested, or otherwise treated to create a solution for final analysis. PTFE filters were not the first choice because of their expense, but they were useful for reactive substances and in cases where the analytical procedure required a solvent that was not compatible with other filter media. For example, when HPLC was the preferred analytical technique, the choice of sampling device, especially the filter, was dependent upon the solvents used in HPLC analysis. The material was usually extracted from the filters in the same organic solvent that was used as the HPLC eluent. MCE filters dissolve in solvents such as methanol and acetonitrile, which are commonly used HPLC eluents. PTFE filters, being very inert, were widely used here.

Generally, any of these filters satisfied the requirements for collection of most of the particulates to be analyzed. However, certain analytes and their associated analytical methods gave better results with alternative filters. The filter cassette was also tested as part of the total sampling device. Some substances were partially collected on the cassette and the substance had to be rinsed from the cassette with solvent to avoid losses.

\section{Sorbents}

The procedure most commonly used for sampling vapors, especially organic vapors, is a glass tube containing two sections of activated charcoal. This tube contains a 100-mg front section for sample collection and a 50-mg backup section for indication, by subsequent analysis, of breakthrough of the front section. Both petroleum-based and coconut-based charcoal have been used in these tubes, along with some other types. For some cases where charcoal was inadequate, silica gel was used as, for example, in sampling amines.

In this program, many of the organic vapors studied were not amenable to collection on charcoal for reasons such as reactivity with the charcoal, irreversible adsorption, or incomplete desorption. Silica gel has also been used extensively in the past; however, for most substances, it has severely reduced collection efficiency under humid conditions. The use of porous polymers as sorbents has been studied considerably in the validation tests. Methods have been developed in these studies using the following sorbents:

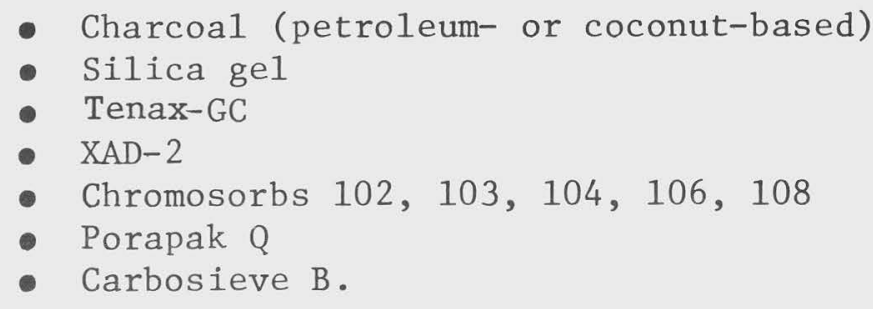


The Tenax-GC, XAD, Chromosorb, and Porapak materials are porous polymers that have surface areas ranging from about 35 to $800 \mathrm{~m}^{2} / \mathrm{g}$. Tenax-GC is a polyphenyl ether, while most of the Chromosorbs, XADs, and Porapaks are cross-1inked polystyrenes. Carbosieve is a high purity synthetic carbon. Sorbent tubes based on these types of media represented the most attractive means of collecting vapors and gases. The sorbent tube is least obtrusive to wear, stores well, and ships reliably. The handling procedures for such tubes are simple and efficient.

In choosing a sorbent for a particular compound, we tried to standardize upon one type of sorbent. However, it was found in early work that significant differences in analyte recovery, capacity, and storage stability can be obtained with different sorbents. Batch-to-batch variation in the same sorbent was also observed. To overcome this, cleanup procedures were established to reduce the effects of residual solvents, unreacted monomers, or other impurities in the porous polymer. The mesh size of the sorbent was a factor in determining the physical dimensions of the sorbent tube. Lower mesh sizes, in the range of 20/50, were desirable since they produced less resistance to air flow. Most personal sampling pumps do not have sufficient capacity to draw more than 1 inch of mercury.

Al1 the sorbents described above are intended to trap analytes by a process of physical adsorption. An alternative set of sampling procedures involves "active" chemisorption, in which the analytes are not only removed from the gas stream being sampled but altered in structure. The advantages of the active type of method are that sorption is frequently more efficient and more specific for the analyte of interest. In addition, the analyte may be converted into a more stable species during the sampling procedure.

Active sorbents developed included Chromosorb 104 coated with maleic anhydride to collect cyclopentadiene, and mercuric cyanide-treated silica gel for collection of phosphine. A triethanolamine-coated molecular sieve was used to collect nitrogen dioxide.

Impinger/Bubbler

Midget bubblers and impingers, filled with either solvent or reactant solutions, were used in this program; however, because of their inconvenience in industrial hygiene applications, these samplers were used only when solid sorbents were ruled out. Since the solutions must be shipped from the field to the laboratory, the choice of solvent was limited to those having a flashpoint at or above $60^{\circ} \mathrm{C}\left(140^{\circ} \mathrm{F}\right)$.

Particulate/Vapor Sampling Methods

The simultaneous collection of vapor and particulate is a difficult problem in personal sampling. The use of a filter/bubbler combination is awkward, and selection of the bubbler solvent is often limited by shipping regulations. A1though some vapors may be collected on filters, this was not found to be quantitative in this program. The problem of storage of a volatile substance on a filter must also be considered.

As discussed earlier, in many instances, it is necessary to generate test atmospheres to determine the physical state of a compound and the collection efficiency of a filter and/or sorbent. The estimation of the vapor/particulate ratio 
may also depend on sample loading. For example, in a short sample time, vapor may be efficiently collected on a filter, but longer term sampling may reveal saturation of the filter with vapor and eventual passage of the vapor into a backup bubbler or sorbent tube.

For substances that exist as both a vapor and a particulate, both a filter and sorbent tube or backup bubbler or impinger were used. Desorption efficiencies were performed for each part of the sampling train and, possibly, for the sampling devices combined. The vapor/particulate ratio was determined at a minimum of one level.

Other Sampling Methods

At present the most acceptable procedure for sampling some stable gaseous materials is collection in bags. These gases are usually too volatile to be collected efficiently with a sorbent tube. Methods were developed and validated for carbon monoxide, carbon dioxide, sulfur hexafluoride, sulfuryl fluoride, methyl acetylene, and methyl acetylene/propadiene mixture using this technique.

GENERAL METHODS

A concerted effort was made to develop methods that could be applied to several substances within the same compound class. This approach was reasonably successful for pesticides, aldehydes, and organolead compounds. The outcome was satisfactory for substances with similar properties; however, special treatment was necessary for single compounds within a class that were unusually more reactive or volatile.

Pesticides

The development of a universal filter/sorbent sampling train for pesticides was an objective of high priority in this program. Where possible, a 37-mm MCE filter followed by a sorbent tube containing 20/40 mesh Chromosorb 102 was used. The filter and sorbent were combined and the pesticide was desorbed with toluene. The resulting solution was analyzed by GC with an appropriate detector. This method was validated for endrin, chlordane, and ronnel. Methods were also validated for phosdrin, TEPP, and heptachlor using a Chromosorb 102 sorbent tube only. Chromosorb 102 was not a satisfactory sorbent for some substances. A method was validated for demeton using an MCE filter/XAD-2 sampling train with the same analytical methodology described above.

When HPLC was the preferred analytical method for a pesticide, the choice of sampling device and sample treatment procedure was affected by the HPLC eluent. PTFE or glass fiber filters were used in methods were solvents such as methanol and acetonitrile were the appropriate extraction solvents or when the MCE filter was not satisfactory. Validated methods include ANTU, 2,4-D, paraquat, pyrethrum, rotenone, 2,4,5-T, thiram, and warfarin.

\section{Aldehydes}

The aldehydes studied in this program include acetaldehyde, chloroacetaldehyde, formaldehyde, furfural, crotonaldehyde, and acrolein. Because of the instability of the aldehydes, they are best collected by derivatizing in the field 
during sample collection. The Girard T derivatives of the aldehydes were studied extensively and methods were validated based upon formation of the Girard $T$ derivative of acetaldehyde, formaldehyde, and furfural. A bubbler containing Girard $\mathrm{T}$ reagent in an aqueous solution at a controlled $\mathrm{pH}$ was used to collect samples. Since the Girard T derivatives are positively charged species that exhibit UV absorptivity, different analytical approaches were feasible.

Polarographic methods of analysis of the derivatives of formaldehyde and crotonaldehyde were developed. Analysis by HPLC was later considered in order to achieve greater resolution between the individual aldehydes and potential interferences. Methods validated using HPLC analysis include acetaldehyde and furfural. Tests indicated that HPLC analysis may be applicable to the Girard T derivatives of other aldehydes such as formaldehyde, propionaldehyde, and benzaldehyde.

Girard $\mathrm{T}$ derivatives of chloroacetaldehyde, crotonaldehyde, and acrolein were not stable or quantifiable. Alternative methods were developed based upon the derivative formed by reaction of crotonaldehyde with hydroxylamine, and the formation of the hydrate of chloroacetaldehyde.

\section{Organoleads}

Methods for sampling and analysis of tetraethyl lead and tetramethyl lead were developed based on collection of XAD-2, desorption with pentane, and analysis by gas chromatography with a photoionization detector. The photoionization detector was used because of a much higher selectivity for organoleads over alkanes than that available with the flame ionization detector. This was necessitated because of the low standards for these compounds.

A larger amount of $\mathrm{XAD}-2$ was required for tetramethyl lead because of its much higher volatility. It is quite likely that both could be collected simultaneously with the larger sorbent tube.

\section{Others}

Attempts were made to develop general methods for mercaptans, amines, alcoholamines, and nitroalkanes. However, results were not satisfactory. A method was developed and validated for n-butyl mercaptan based upon collection with a sorbent tube containing Chromosorb 104, and analysis by gas chromatography. The sorbent did not have adequate capacity to collect methyl or ethyl mercaptan. The amines and alcoholamines were difficult to analyze by GC, the preferred method which incorporated derivatization of the collected sample. Sensitivity and precision were very poor for some amines. Ion chromatography proved a more efficient and sensitive analytical method than GC for methylamine. The nitroalkanes gave inconsistent storage stability results on various solid sorbents.

\section{LABORATORY TESTS}

\section{Calibration Curve}

Once an analytical method was selected, optimum conditions were established to achieve maximum selectivity and sensitivity of the compound of interest. For example, in GC or HPLC the analyte must be well separated from the solvent peak 
so that electronic integration is possible and that a reasonable retention time is acheived to minimize analysis time. The linear range of the detector was established. If the instrument response was nonlinear, such as with the flame photometric detector for sulfur, a wider range and increased number of standards became necessary to determine the instrument response. A calibration curve was then prepared over the range of 0.1 to 3 times the OSHA standard for the proposed sample volume. An estimate was made of the lower detection limit. Similar aspects for spectroscopic methods were also considered.

The effects of substances that may significantly interfere in the analysis of the analyte were also investigated. Mixtures of the suspected interferences and the analyte were prepared at concentrations representative of expected atmospheric concentrations and analyzed. If necessary, adjustments were made in analytical conditions to eliminate or minimize interference effects.

Analytical Recovery Tests

The sampling medium was selected by testing various media (sorbents and filters) and desorption or extraction solvents. Samples were spiked with analyte, allowed to equilibrate, and desorbed. The combination of sampling medium/solvent yielding the most promising results was tested further, unless general methodology was applied. For sorbents, the next step was to determine their breakthrough volume at high humidity, as described in the next section. Once capacity was demonstrated and the sampling flow rate and time established, more extensive analytical recovery tests were performed.

Three sets of six samples were evaluated. For these tests, the sampling medium was spiked to represent samples taken at 0.5, 1 and 2 times the OSHA standard for the selected sample volume. For sorbents, stock solutions of the analyte in an inert solvent were prepared at such concentrations that less than 10microliters of solvent was necessary to apply the analyte to the sorbent.

After equilibration of the material on the sorbent, the analytical recovery was determined. Standards were prepared and analyzed at the same time as the desorbed samples. A minimum recovery of $75 \%$ with a relative standard deviation of no more than $7 \%$ for six samples was required before proceeding. A recovery of greater than $90 \%$ was preferred.

Analytical recovery tests for filters were performed in a manner similar to that for sorbents. Recoveries from filters should be quantitative ( $100 \%)$.

These tests were expected to show that recovery was either constant or increased with the amount of analyte used. After these tests were completed, a graph of recovery or desorption efficiency (amount recovered divided by the amount spiked) as a function of the amount of spiked substance was constructed for later use in the analyses of generated samples.

For a filter/sorbent combination sampler, recoveries from both filter and sorbent were evaluated. Generally, the recovery from both collectors was evaluated by spiking with amounts of analyte representing samples collected over the range 0.1 to 2 times the OSHA standard. Determinations over this extended range were necessary since the vapor/particulate ratio in field samples may differ from that in laboratory tests. Satisfactory recoveries $(>90 \%)$ must be 
obtained for each combined collector. If the filter and sorbent are combined and analyzed together, the applied recovery correction must be statistically the same for both filter and sorbent over the entire range.

Determination of Breakthrough Volume or Collection Efficiency

Breakthrough Volume--

To evaluate a sorbent for breakthrough volume, test atmospheres of the analyte were generated in air of at 1 east $80 \%$ relative humidity. The details of the test atmosphere generator and specific generation techniques are given later in this section. The sampling rate was based on the pressure drop across the sampling device, the minimum length of time required for sampling, and the expected capacity based on past experience.

The test atmosphere was generated at a concentration twice that of the OSHA standard. For this determination, the time of appearance of the analyte in the effluent from the front section of the sampling device was monitored.

When possible, online monitoring was used. A set of six samplers was placed in the sampling chamber, and the concentration of the test atmosphere was measured. During sampling, the effluent from the sorbent tubes was monitored and compared with the test chamber concentration. The $5 \%$ breakthrough volume is defined to be when the effluent concentration reaches $5 \%$ of the influent concentration. The test was continued until the effluent concentration was at least one-third of the influent concentration so that premature breakthrough of one tube due to "channeling" was not mistaken for overloading of the six tubes.

Online detection of the low levels of many substances studied in this program was not possible. In this case, as when the generated concentration was below the detection limit of the monitor, the capacity test was conducted using a number of samplers, each containing a backup section. After sampling began, the samples were removed, one by one, at regular intervals, and each section analyzed separately to determine when the amount of material in the backup section was equivalent to $5 \%$ of the amount in the front section.

Once the breakthrough volume was determined, a sampling volume no greater than two-thirds of the breakthrough volume was recommended. A minimum one-hour sampling period was imposed for TWA determinations.

If the breakthrough volume was sufficient for collection at twice the OSHA standard at the recommended flow rate and sampling period, method testing was continued. If not, appropriate steps were taken to improve the method. Reduction in the sampling rate, increased sorbent volume, and selection of another sorbent were options.

The testing of filter/sorbent collection media was similar to the procedure described above for sorbents alone. However, careful observation was made of the amount collected on filter and sorbent individually over time. Multiple samplers were used in the capacity tests, and samplers were removed and analyzed at regular intervals throughout the test. The amounts collected on the filter, sorbent, and backup sorbent were plotted versus time. The plot frequently indicated the extent of vapor collected on the filter. It also gave a much 
better indication of the ratio of particulate to vapor than any test over one time interval.

Collection Efficiency--

In evaluating the efficiency of particulate collection by a filter, test atmospheres were generated as described above for the testing of sorbent capacity. Six collectors with appropriate backup collectors (filters and/or bubblers) were tested long enough for the substance of interest to be detected on the backup collector, if present. If a detectable amount of the analyte were found in the backup collector, the sampling device was modified to include a backup collector or an alternate collecting medium was selected. If the presence of vapor were a possibility, the test atmosphere concentration was at the OSHA standard or lower because vapor would be more prevalent at the lower levels. Extended sampling periods were sometimes necessary to obtain enough sample for determination. Sufficient sample was collected so that an amount equivalent to $5 \%$ to $10 \%$ of a sample collected at 0.5 times the OSHA standard could be detected on the backup collector. This was to ensure that an unacceptable bias did not exist that would invalidate the method.

Generation and Collection of Samples

Test atmospheres were generated at three known concentration levels. A set of six samples was collected from each test atmosphere and analyzed by the prescribed analytical method. Test atmosphere concentrations at $0.5,1$, and 2 times the OSHA standard were generated (within 25\%). Samples were collected at the recommended flow rate and sampling period. This part of the validation procedure tested the overall sampling and analytical method for precision and accuracy.

Independent Method of Determining Test Air Concentration

For most of the materials in this program, the determination of a "true" test air concentration constituted a significant part of the study. Direct measurement was the obvious method of choice, but this was not feasible for many substances. Determination of nonreactive gases and vapors was accomplished using the techniques of delivery rate calibration, such as syringe drive and permeation tubes.

The measurement of reactive gases and vapors, not suitable for direct determination by use of delivery rate information, was often achieved by collecting and analyzing them by an accepted alternative method, frequently one not amenable to industrial hygiene sampling. For example, collecting parallel samples on a solid sorbent and in a bubbler, followed by analysis by GC and HPLC allowed assessment of the accuracy of the method under test. However, confidence in the alternative method first had to be established by appropriate testing.

The determination of test concentrations of dusts, aerosols, and especially mixtures of both aerosol and vapor was considerably more difficult. The choice of an independent method for these materials must be made carefully. Use of a less reliable method to assess accuracy may result in failure to validate an acceptable procedure. The method most often used for these compounds was to use an alternative method of collection and compare the results with the results obtained by the validation method. This was most frequently used for particulates 
where parallel samples were collected on different filters. For particulate/ vapor mixtures, when the proposed method used a filter and sorbent, a different type of filter followed by a bubbler was used to sample from the same atmosphere. The alternative method was also thoroughly tested, using a reasonable number of samples. Because this technique relied heavily on good collection efficiency, all the sampled material was accounted for. It was assumed that there was no discrimination of particle sizes collected resulting from different inlet velocities of the two samplers.

\section{Storage Stability of Generated Samples}

To determine if collected samples can be stored for 1 week before analysis, 12 samples were collected simultaneously from a test atmosphere at the OSHA standard level. Six samples were analyzed immediately after collection and the remaining six were stored on the laboratory bench for 7 days. The average concentration determined from the six samples analyzed immediately was compared to the average concentration determined from the six samples analyzed after 7 days storage.

At times the storage stability of the collected sample was a critical factor in the method. If poor stability was suspected, tests were conducted early in experimental studies to determine corrective measures.

Method Validation

For a sampling and analytical method to be validated, certain requirements based on statistical criteria must be met. The criterion for a valid method was based on whether the overall sampling and analytical result could come within $25 \%$ of the "true" value at the $95 \%$ statistical confidence level for any individual sample. A detailed discussion of the statistical protocol for this program and the definition of terms are in Appendix B. To meet this requirement, an unbiased method must have a coefficient of variation (CV) of less than 0.128 . Taking into account random errors of estimation, the total CV from laboratory validation data (with 15 degrees of freedom) should not exceed 0.105. This includes a maximum allowable imprecision of $5 \%$ due to the use of a personal sampling pump. A biased method must meet more stringent requirements. A summary of the criteria that were used to evaluate the test method is listed below:

- Analytical recoveries

- Capacity

- Storage stability
Three sets of six samples were spiked at 0.5 1 , and 2 times the OSHA standard for the recommended sample volume. Recovery must be at least $75 \%$ (preferably $>90 \%$ ).

At least six determinations were made at twice the OSHA standard at greater than $80 \%$ relative humidity (for sorbents). The recommended sampling volume must be less than two-thirds the breakthrough volume.

12 samples were collected from test atmospheres at the OSHA standard; 6 samples were analyzed immediately, 6 after 7 days storage. Means of concentrations found for each set must agree within $10 \%$. 
- Precision

- Accuracy
1. Three analytical recovery sets (above). The total CV pooled from three groups of 6 samples must be less than 0.07 .

2. Three sets of six samples each were collected from test atmospheres.

The total CV pooled from three groups of six samples must be less than 0.105 (including allowance of 0.05 for error due to the sampling pump.

Three sets of six samples were collected from test atmospheres. The mean concentration determined by the test procedure must be within $10 \%$ of the "true" concentration at each of three test levels. In addition, precision and uncorrected bias must be such that, in combination, they do not exceed the $25 \%$ rule stated above (see Figure B-1, Appendix B).

These are the minimum criteria used to determine acceptability. In addition, the method for each compound was evaluated for possible defects that might occur in field sampling situations, either by additional laboratory testing or by reference to literature.

Bartlett's test for homogeneity of variances was applied to the coefficients of variation at $0.5,1$, and 2 times the OSHA standard for samples collected from test atmospheres. If Bartlett's test was passed, it was feasible to pool the coefficients of variation to calculate $\overline{\mathrm{CV}}_{\mathrm{T}}$. All data were subjected to Grubb's test to determine if one point in a set of data should be rejected as being an out1ier.

The precision of the analytical method was assessed using the analytical recovery data. The pooled coefficient of variation of $\left(\overline{\mathrm{CV}}_{1}\right)$ was calculated.

Precision and accuracy of the total sampling and analytical method were evaluated using the results from three sets of analytical recovery tests, the three sets of samples collected from test atmospheres, the breakthrough tests, and the storage stability tests. The pooled coefficient of variation $\left(\overline{\mathrm{CV}}_{2}\right)$ for the three sets of samples collected from test atmospheres was calculated. To obtain a measure of the accuracy of the method, the mean value of the concentration found by analysis at each level was compared with the value for the "true" concentration determined by the independent method.

The total coefficient of variation $\left(\overline{\mathrm{CV}}_{\mathrm{T}}\right)$ was calculated using the pooled $\overline{\mathrm{CV}}_{1}$ for three sets of analytical samples and the pooled $\overline{\mathrm{CV}}_{2}$ for the three sets of samples collected from test atmospheres. 
General

Extensive facilities for preparing synthetic atmospheres were developed at SRI and ADL for use on this program. These facilities were successfully used for validating methods to sample and analyze gases, vapors, dusts, aerosols, and fumes. They consisted basically of a source generator, which introduces test air containing high analyte concentration into a dilution system, where three concentration levels are automatically prepared and delivered to sampling chambers. The two main dilution systems are described in this section.

Generation of test atmospheres by dynamic means was used because of the advantages over static methods. Wall losses through adsorption, which may be a significant problem in static systems, become negligible in dynamic systems because the apparatus walls eventually equilibrate with the gas stream. Dynamic systems are especially appropriate for producing mixtures of compounds subject to decomposition because the labile products are continuously swept away and the mixture is continuously replaced.

In addition to the main generation facilities, many special systems were designed and constructed for use with reactive compounds. Separate systems constructed exclusively of Teflon and glass were used with reactive substances including fluorine, mercury, and several amines. Each substance in this program was generated from a specific source generator, depending on its physical state and whether it existed as a vapor and/or as an aerosol in the atmosphere at the desired concentration. Details of the specific source generators used for individual substances appear in the Backup Data Report for the substance.

Methodology

Aerosols--

Test atmospheres containing aerosols may be conveniently generated by a number of techniques including:

- Spray drying

- Atomization

- Condensation

- Thermal decomposition

- Dust dispersion.

Spray drying--Spray drying is applicable to substances that are soluble in a convenient solvent. This method involves atomizing a solution to form a mist. Larger particles are removed by impaction. The remaining flow is mixed with solvent-free air to permit evaporation of solvent from the droplets. Residue particles of the solute are left behind as the solvent evaporates.

The size of the aerosol particles produced by spray drying is related to the initial mist particle size by

$$
\mathrm{D} \propto \mathrm{C}^{1 / 3} \mathrm{D}_{\mathrm{m}} \text {, }
$$


where $\mathrm{D}$ is the average particle size of the aerosol, C is the volume concentration of solute in the solution (volume of solute per unit volume of solution), and $\mathrm{D}_{\mathrm{m}}$ is the average size of the mist droplets. Small pneumatic atomizers, which are commonly used for the laboratory production of aerosols by the spray drying technique, usually produce mists with mean diameters of 1 to 10 microns. Consequently, for a one part per million solution (volume/volume basis), such atomizers will produce particles in the hundredth micron size range.

The concentration of aerosols produced by the spray drying method may be varied by varying the concentration of the solution. The relation between solution concentration and aerosol concentration at fixed volume production rate is roughly linear over a wide range of concentration. Aerosols in the concentration range of $\sim 0.001$ to $\sim 10 \mathrm{mg} / \mathrm{m}^{3}$ may be easily produced by spray drying. The spray drying technique has been used for most of the aerosols generated in the validation program, including the majority of the pesticides.

Atomization--Liquid aerosols and aerosols of readily melted solids may be generated by direct atomization using, for example, a pneumatic nebulizer such as the Collison. When a pneumatic atomizer is used, the aerosol condensation may be varied by changing the pressure of the atomizer air supply pressure. The mass median diameter of the aerosols produced by the small pneumatic nebulizer is typically in the micron range. In this program, the direct atomization method was applied to dimethylphthalate, a liquid, and dibutyl phosphate, a low melting solid.

Condensation--The condensation method requires the formation of a vapor-air mixture that is supersaturated with respect to the vapor. Condensation from the supersaturated vapor may then occur either on foreign particles present in the gas (heterogeneous nucleation) or on small clusters of vapor molecules that are formed at random (homogeneous nucleation).

Supersaturated vapor mixtures are usually formed by passing air or an inert gas over a heated pool of liquid (or over a heated solid) so that the stream may pick up vapor. The vapor-laden stream is then cooled, usually by mixing with a cooler gas stream or by thermal conduction so that the partial pressure of the vapor rises above the saturated vapor pressure.

The concentration of the aerosol produced by the condensation method is, of course, determined by the partial pressure of the vapor in the supersaturated stream. The partial pressure, and thereby the ultimate aerosol concentration, may be varied by varying the temperature of the vapor source. Particle size is determined by several factors: the number of nuclei present, the degree of supersaturation achieved, and the rate of change of the degree of supersaturation. These factors are independent. Particles ranging from about 0.01 to more than 10 microns have been prepared by this method. Although it is best known as a method for generating monodisperse aerosols, condensation may also be used to produce polydisperse aerosols. Dinitro-o-cresol was generated using this method.

Thermal decomposition--Thermal decomposition methods may be used to prepare metal oxide fumes. An aerosol of a precursor to the metal oxide--i.e., a substance that is readily decomposed, thermally, to yield the oxide--is first generated and then is heated by passing it through a heated tube to decompose it to the oxide. Metal formates, oxalates, and the like, which readily yield the 
oxides and which do not produce objectionable side products, are commonly used precursors. In this program, fumes of iron oxide, vanadium oxide, and copper oxide were generated using this method.

Dust dispersion--Aerosols of mineral particles and a few other materials may be prepared by dust dispersal techniques. A number of dust dispersal devices have been constructed. A commonly used one is the Wright Dust Feeder. In this device the dust is compressed into a cylindrical compact, which is placed in an electrically driven mechanism that rotates it and drives it against a fixed knife blade at a fixed rate. Material is scraped off the compact by the blade, entrained in an air stream, and blown against a metal plate to break up particle aggregates.

For the dust dispersal method to be used, it must be possible to obtain the material of intent as a finely divided powder. In addition, for the Wright Dust Feeder to be used, it must be possible to prepare a dispersable compact of the powder. Soft materials that compress into a solid compact in which the individual particles are merged obviously cannot be dispersed by this method.

The concentration of the dust produced by the Wright mechanism is varied by varying the rate of advance of the dust compact. Particle size, in principle, at least, is the same as the size of the particles in the initial powder; however, in practice complete breaking up of aggregates may not occur. This method was used to generate dusts of warfarin, zinc oxide, rotenone, and tantalum.

Gases and Vapors--

Test atmospheres of gases and vapors are generally prepared dynamically by the following methods:

- Dilution

- Syringe injection

- Vaporization

- Permeation

- Chemical reaction.

Dilution--For gases, the simplest method of introduction into the system is dilution. A cylinder of the compressed gas is fitted with a metering apparatus (regulator and/or critical flow orifice) and the effluent stream injected directly into the dilution system. The flow rate of the analyte can be calibrated and the concentration of each test atmosphere can be calculated with the known dilution air flows. Most nonreactive gases were generated by this technique.

Syringe Injection--Injection of a volatile liquid at a known rate into an air stream of known flow rate is a convenient method for producing vapor mixtures of known composition. Syringe drives may be used to inject liquids or gases at constant rates as low as a few microliters per minute. This method may be used to prepare mixtures of nonreactive compounds, with concentrations of parts per million to the fractional percentage level. Since the rate of delivery of the syringe drive may be accurately determined by weighing the amount of liquid delivery over a known period of time and from measuring the dilution air flow rate, very good accuracy may be obtained by this method. The syringe injection technique has been used for many vapors in this program. 
Vaporization--The vaporization method simply involves contacting a flowing air stream with a liquid or solid so that the stream may pick up vapor from the condensed phase. Typically, gas dispersion bottles are used to produce vapors of 1iquids. More complex equipment must be used to produce completely saturated gas streams. Temperature and flow rate over the condensed phase may be used as control parameters for varying the vapor concentration.

The vaporization method is applicable from the parts-per-billion level to several percent concentration range. Usually, mixtures prepared by this method are analyzed by an alternative analytical method to determine concentration rather than being computed from vapor pressure data.

Permeation--Permeation tubes offer a third method for the preparation of vaporair mixtures of known concentration. The substance of interest is sealed in liquid form inside a tube of some polymeric material, such as Teflon. Vapor produced by the liquid permeates through the walls and is picked up by an air stream passing over the tube.

The quantity of vapor delivered by a permeation tube, $q$, is given by

$$
q=\operatorname{DSA}\left(\frac{\mathrm{P}_{1}-\mathrm{P}_{2}}{\mathrm{~L}}\right)
$$

where $\mathrm{D}$ is the diffusion constant of the vapor through the polymer, $\mathrm{S}$ is the solubility constant of the gas in the polymer, $A$ is the surface area of the tube, $\mathrm{P}_{1}$ and $\mathrm{P}_{2}$ are, respectively, the total pressures on the outside and inside of the tube walls, and $\mathrm{L}$ is the wall thickness. Because $\mathrm{D}, \mathrm{S}$ and $\mathrm{P}$ are temperature dependent, the rate of delivery can be varied by varying the temperature of the tube. Usually the concentration of the vapor mixture produced by this technique is determined from the weight loss of the tube and the flow rate of the stream passing over it.

The permeation tube methods are used to prepare mixtures with concentrations in the part per billion to hundreds of parts per million range. Dimethylmercury was generated using a permeation tube for validation of the alkyl mercury method.

Chemical reaction--Chemical reaction methods are convenient for very reactive substances not readily available in a stable form. Two examples are

(1) generation of stibine by injection of an aqueous solution of potassium antimony tartrate and sodium borohydride into dilute sulfuric acid, and

(2) preparation of cyclopentadiene by passing the dimer through a tube furnace.

Main Dilution/Sampling System

SRI Internationa1--

The system for generating and collecting samples of vapor, inorganic/organic particulate, dusts, and fumes consists basically of a sample generator, a mixing and dilution section, and three sampling chambers. Samples are generated at a concentration 2 times the OSHA standard, serial dilutions are made to 1 and 0.5 
times the standard, and samples are collected simultaneously at the three concentrations. A schematic of the generation system and associated components is presented in Figure 2. A photograph of the system is in Figure 3.

The generation system is large enough to be used for polydispersed aerosols as wel1 as for gases and vapors. The primary dilution chamber is 48 inches by 4 inches and may handle air flows up to $400 \mathrm{~L} / \mathrm{min}$. The large volume dilution chamber is important for several reasons. Even at high air flow rates, the velocity of particles is low to allow complete solvent evaporation in the generation of aerosols. The air velocity is also low enough to avoid impaction on the walls while great enough to prevent particle diffusion to the walls. For these same reasons, the sample rationing system is only 1 inch in diameter and handles a flow of only $52 \mathrm{~L} / \mathrm{min}$. Gravitational settling is avoided by maintaining a sufficient air velocity.

The sampling cones for the three chambers are 6-inch I.D. at the base (point of sample collection) and narrow to 1 -inch $\mathrm{I}$.D. at the point of attachment to the sample rationing system. A constant total air flow of $26 \mathrm{~L} / \mathrm{min}$ through each cone causes a gradual reduction in aerosol velocity toward the point of sample collection. The air velocity at the collection point is $2.4 \mathrm{~cm} / \mathrm{sec}$.

All portions of the generation system that come in contact with the test atmosphere are constructed of stainless steel or Teflon to avoid contamination. Sections of the generation system at which dilution air is added are constructed such that the incoming air forms a "high-velocity sheath" around the air/analyte mixture that is to be diluted. This sheath serves two functions. The dilution air sheath becomes increasingly less coherent and stable as it moves downstream of its point of entrance and hence is turbulently mixed with air/analyte test atmosphere. At the point of entrance of the dilution air stream, a Venturi effect accelerates the air/analyte mixture to a high velocity. The dilution air sheath also prevents interaction of the accelerated air/analyte stream with the walls of the chamber, thus eliminating a large source of aerosol loss by impaction.

The system being used to generate the initial concentrations of vapor, gas, or particulate is interfaced with the dilution apparatus at the primary dilution chamber. The output of the generator is diluted with the appropriate amount of air to obtain a concentration 2 times the OSHA standard. Of the total amount of material generated at twice the level, a flow of $52 \mathrm{~L} / \mathrm{min}$ enters the rationing system. Under control of a vacuum exhaust orifice, material at 2 times the level enters the first sampling chamber at a rate of $26 \mathrm{~L} / \mathrm{min}$. Downstream of the entrance to the first sampling chamber, dilution air is added (via a critical orifice) at a rate of $26 \mathrm{~L} / \mathrm{min}$. Thus, the flow of material at 2 times the level that did not enter the first sampling chamber $(26 \mathrm{~L} / \mathrm{min})$ is diluted with air at a flow rate of $26 \mathrm{~L} / \mathrm{min}$ to a final concentration of 1 times the OSHA standard level. Analyte at the 1 times level then enters the second sampling chamber at a rate of $26 \mathrm{~L} / \mathrm{min}$. The remaining flow, $26 \mathrm{~L} / \mathrm{min}$ is diluted again with air at $26 \mathrm{~L} / \mathrm{min}$ to achieve 0.5 times the OSHA standard level. The analyte/air mixture at the 0.5 times level is drawn into the third sampling chamber at $26 \mathrm{~L} / \mathrm{min}$. The remaining material in the rationing system not drawn into the sampling chambers is removed at a rate of $26 \mathrm{~L} / \mathrm{min}$ by the fourth critical orifice in the vacuum exhaust system. This removal of test atmosphere volumes and addition of 


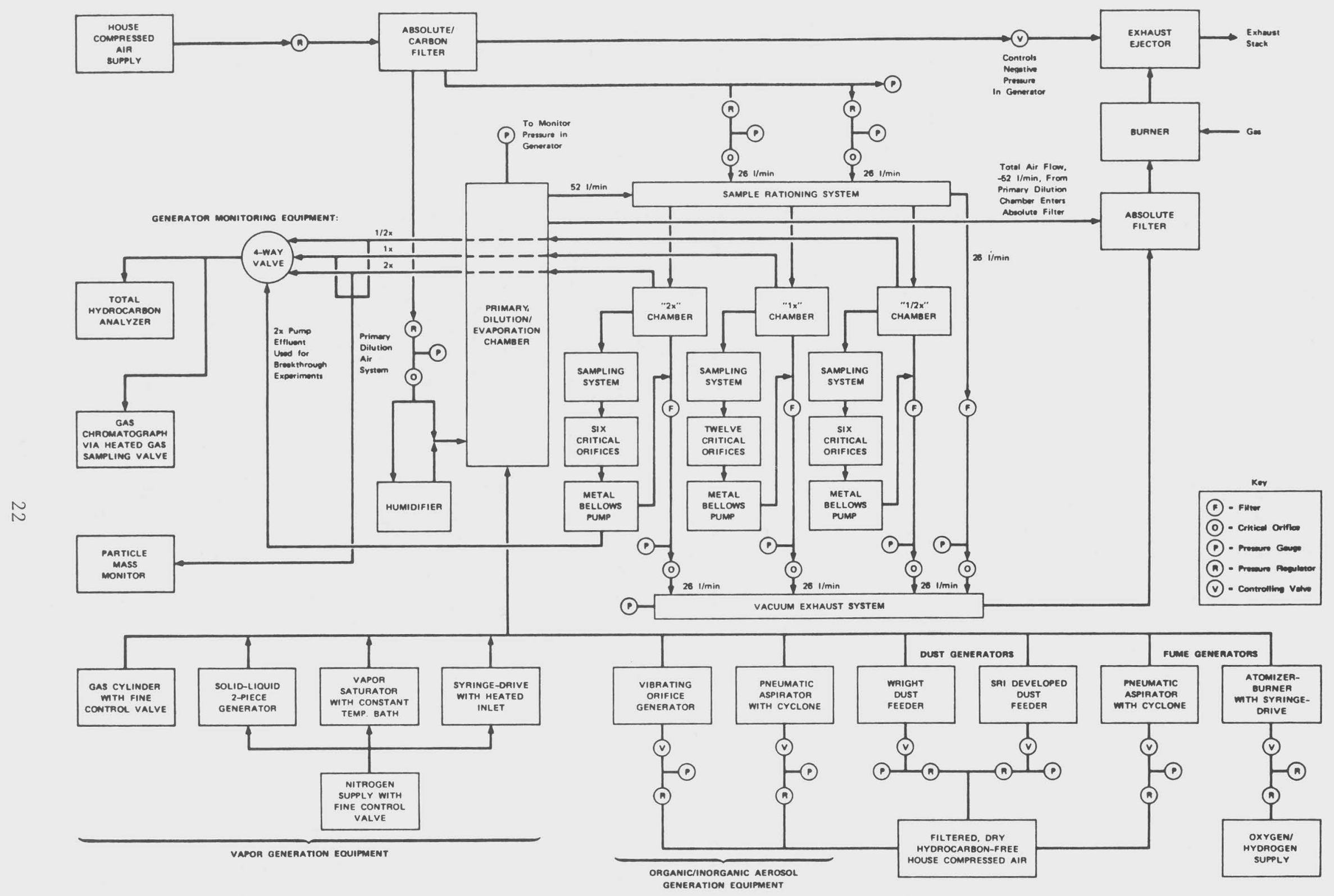

FIGURE 2 SRI GENERATION/DILUTION/SAMPLING SYSTEM 


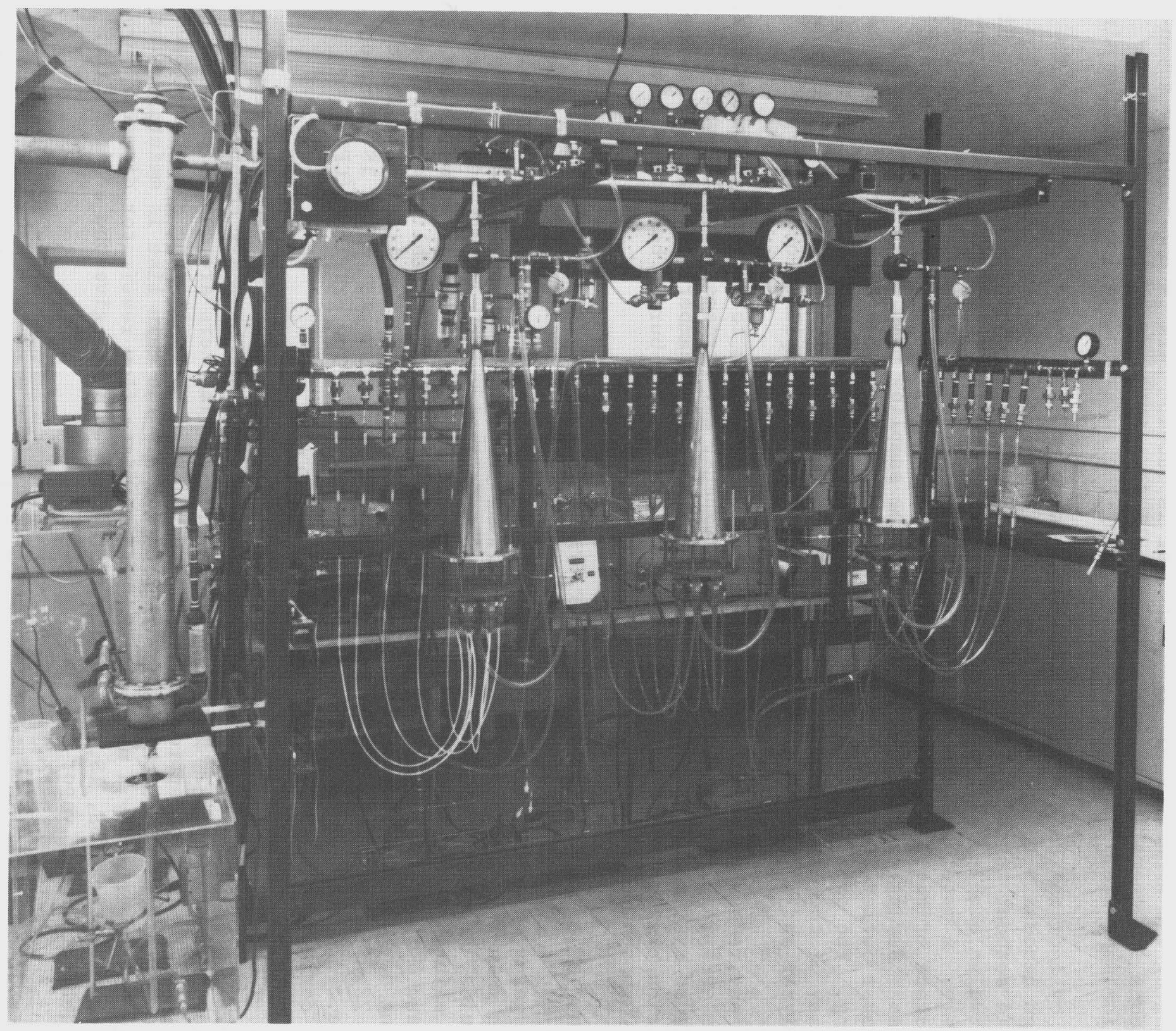

FIGURE 3 SRI SAMPLE GENERATION SYSTEM FOR VAPORS AND PARTICULATES 
measured volumes of air thus achieves serial dilutions to 1 times and 0.5 times the OSHA standard level.

The dilution ratios from chamber to chamber can also be varied by simply changing the amount of dilution air that is added. This is particularly advantageous in generating aerosols, where wall deposition of particles in the rationing system can be offset by changing the rate of addition of dilution air.

The cylindrical section at the base of each sampling chamber contains the fittings necessary to collect samples, using any of a variety of sampling media-solid sorbent tubes, filters, liquid scrubbers, or a combination of these. Six to twelve samples each at three concentration levels can be collected simultaneously. A metal bellows vacuum pump is used for sampling from each chamber. Separate critical flow orifices are used for each sample. Air taken from the chamber during sampling is returned via the sampling pump exhaust line to the chamber outlet line, thus preserving the proper air flows during the time of sampling. The sampling rate therefore does not affect the concentration of material in any of the chambers. All three chambers can be monitored with a hydrocarbon analyzer and a gas chromatograph fitted with a gas sampling loop.

The entire system is maintained at 1-inch water vacuum to prevent toxic materials from escaping into the laboratory. All exhaust air streams (from the vacuum exhaust system and excess from the primary dilution chamber) are fed into a combustion chamber where all toxic materials present are burned before entering the atmosphere.

Arthur D. Little, Inc. Gas and Vapor System---

The vapor generation/dilution system used for the validation studies of vapors and gases, is shown schematically in Figure 4. The system basically consists of a main line air stream to which are added predetermined amounts of various liquids, gases or aerosols to generate the desired vapor concentrations. From the main line, three dilution arms branch off in which the desired multiples 0.5 , 1 , and 2 times the OSHA standard concentration level are established. Six sampling devices are connected in parallel to the $0.5 \mathrm{~S}$ dilution lines and six to the $2 \mathrm{~S}$ dilution 1 ine; twelve sampling devices are connected to the $1 \mathrm{~S}$ dilution line. All these devices are connected via critical flow orifices (CFOs) to the corresponding vacuum lines.

Air flow rates through the system are established by means of CFOs and flow restrictors. The primary air system derived from the house air compressor is maintained at $20 \mathrm{psig}$. The appropriate orifice diameters are chosen to maintain an air flow of approximately $0.1 \mathrm{cu} \mathrm{m} / \mathrm{min}$ in the main line and an addition of $0.05 \mathrm{cu} \mathrm{m} / \mathrm{min}$ to each of the dilution lines. The main line is maintained at $8 \mathrm{~cm} \mathrm{H} \mathrm{H}_{2} \mathrm{O}$ pressure by a needle valve. Appropriate flow restirctor diameters are chosen for the $0.5 \mathrm{~S}, 1 \mathrm{~S}$, and $2 \mathrm{~S}$ dilution lines so as to give the desired final concentrations of vapor in air.

The system was designed to generate either 4 times or 2 times the OSHA standard concentration in the main line. When a 4 times level is generated, $0.05 \mathrm{cu} \mathrm{m} / \mathrm{min}$ of dilution air is added to each dilution line. Orifices are selected so that the $0.5 \mathrm{~S}, 1 \mathrm{~S}$, and $2 \mathrm{~S}$ lines have flows equal to approximately $0.007,0.017$, and $0.050 \mathrm{cu} \mathrm{m} / \mathrm{min}$ respectively of the main line concentration added to the dilution air, thus giving the desired final concentrations. When a main line concentration 


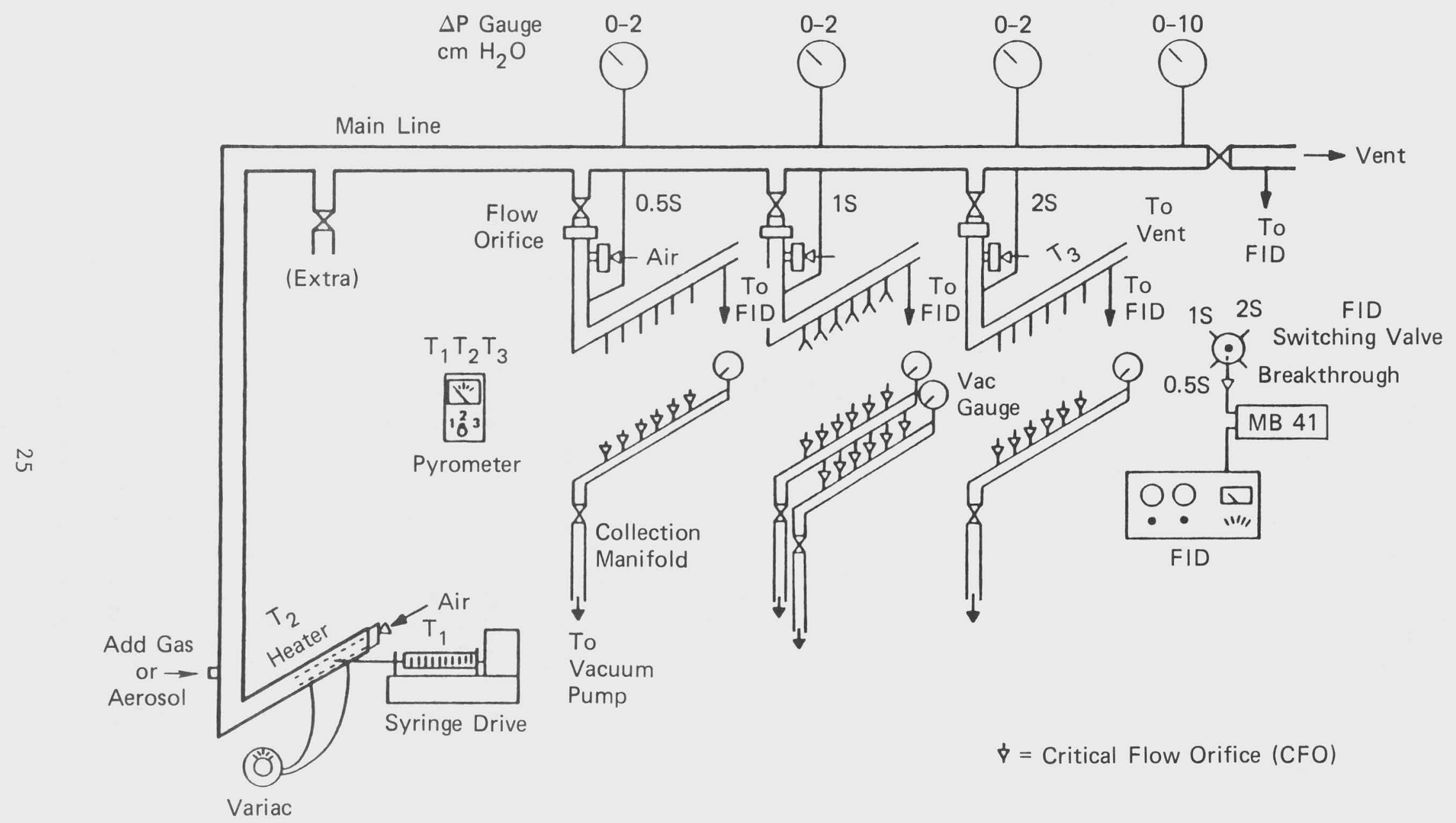

FIGURE 4 ADL VAPOR GENERATION/DILUTION/SAMPLING SYSTEM 
of 2 times the OSHA standard is generated, no dilution air is added to the $2 \mathrm{~S}$ dilution line--0.017 cu m/min is simply allowed to flow through this line--and $0.050 \mathrm{cu} \mathrm{m} / \mathrm{min}$ of dilution air is added to the $0.050 \mathrm{cu} \mathrm{m} / \mathrm{min}$ and $0.017 \mathrm{cu} \mathrm{m} / \mathrm{min}$ of main line mixture admitted to the $1 \mathrm{~S}$ and $0.5 \mathrm{~S}$ dilution lines, respectively.

All materials which the vapor may contact before collection are 316 and 304 stainless steel. A glass heater is included where the liquids are added to the main line. Shutoff ball valves are placed in the dilution lines to allow their independent operation and the calibration of air flows. The main line has a $2.54-\mathrm{cm}$ ( $1 \mathrm{in})$ 0.D., and the dilution lines are $1.90-\mathrm{cm}(0.75 \mathrm{in}) 0 . D$. Diameters were chosen to give turbulent flow with an approximate minimum Reynolds number of 3000 .

Air supply--Air from the house compressor is treated by passing it sequentially through a cotton filter, a silica gel bed, a charcoal bed, and a high efficiency glass fiber filter for removal of water, hydrocarbons and particulate. This air is then connected to a manifold containing six takeoff ball valves. The pressure (20 psig) at the manifold is maintained with a Nullmatic Moore $40 \mathrm{H} 50$ regulator and monitored with an Ashcroft 0-60 psig test gauge. The air supply is used for each of the dilution system connections as well as for the flame ionization detector monitor flame and "zero" air.

Sample collection manifold--Sample flow through the sampling devices connected to the dilution lines is established by connecting each device by means of a short piece of flexible tubing to a CFO which is connected to a $1.27 \mathrm{~cm}$ ( 0.5 in) 0.D. vacuum manifold. Each dilution line has a separate manifold which derives its vacuum from a Model 0322 Gast vacuum pump. The orifices are jewel orifices pressed into a threaded Teflon rod. One end of the rod is screwed into a tee on the manifold, and the other has a hose tabulation fitting connected to it. The orifice is protected from plugging by a piece of 100-mesh stainless steel screen.

Vent System--All excess vapor-laden air is collected via a $3.81-\mathrm{cm}$ (1.5 in) PVC manifolding system where it is passed through a $0.3 \times 0.3 \times 0.6-\mathrm{m}$ charcoal bed. Flow is established by means of a pressure blower on the exit side of the charcoal bed, and it is vented to the laboratory hood exhaust.

Monitors--To provide a ready check on operating conditions, several gauges or monitors have been included in the system. Dwyer Magnehelic gauges monitor the pressure on the main line and each of the dilution lines. A $0-10 \mathrm{~cm} \mathrm{H}_{2} \mathrm{O}$ gauge is used on the main line (setpoint $8 \mathrm{~cm}$ ) and $0-2 \mathrm{~cm} \mathrm{H} \mathrm{H}_{2} \mathrm{O}$ gauges are used for the dilution lines. The purpose of these latter gauges is to provide a check against possible back pressure developing in these lines which would affect the dilution ratios.

The flame ionization detector (FID) is used to determine the time at which the main line concentration has reached equilibrium and to monitor the concentration level during breakthrough studies and sample collection.

Arthur D. Little, Inc. Aerosol System--

The dilution and sampling system used to produce the appropriate aerosol test atmospheres is shown schematically in Figure 5. Basically, the system consists of a main horizontal line into which aerosol and dilution air are introduced and three vertical dilution and sampling sections which branch off the main line. 
These branches are designated as A, B, and C. The dilution and sampling branches $A$ and $C$ are identical and each is equipped with a sampling manifold with six sample ports. The dilution and sampling branch B, on the other hand, is equipped with a sampling manifold with fourteen sample ports. Figure 6 and 7 show these dilution and sampling branches in more detail.

Aerosol dilution ratios in the system are fixed by critical flow orifices. Usually an aerosol with a concentration twice the OSHA standard is prepared in the main line, and this aerosol sampled without dilution in one dilution/sampling section and diluted twofold and fourfold in the other two sections. Other dilution modes may be accommodated simply by changing the critical orifices.

Aerosol dilution occurs in the Teflon venturi-shaped inserts shown in Figures 6 and 7. Dilution air is injected radially into the venturi throat. The quantity of dilution air introduced is fixed by a critical orifice which is connected to one of the constant pressure air manifolds, as described in the section on air supply below.

Isokinetic sampling probes, six for branches $\mathrm{A}$ and $\mathrm{C}$, and fourteen for branch $B$, are located approximatey 30 centimeters downstream of each diluter. The probes convey aerosol to sample collectors (filter cassettes) mounted radially around the outside of the sampling section. There is a luer fitting on each sample port to mate with the filter cassette. Sample flow rate is fixed at $1.5 \mathrm{~L} / \mathrm{min}$ by critical orifices (sapphire orifices supplied by Richard H. Bird and Co., Waltham, MA) mounted on the sample manifold (Figure 5).

As is indicated in Figure 5, a critical orifice is located downstream from the sample probes in each dilution/sampling branch. This orifice is protected from contamination by a Filterite high efficiency filter. For the dilution/sampling branches $\mathrm{A}$ and $\mathrm{C}$, there are seven outwardly flowing streams in each branch--one stream is that which flows through the orifice mentioned above (flow rate $\mathrm{Q}_{\mathrm{T}}$ ) and the other six are the sample streams (total flow rate $Q_{S}$ ) for the dilution/ sampling branch $B$, the flowing streams consist of one which flows through a critical orifice downstream from the sample probes (flow rate $\mathrm{Q}_{\mathrm{T}}$ ) and fourteen from the sample streams (total flow rate $Q_{S}$ ). There are two inflowing streams--the dilution air stream (flow rate $Q_{D}$ ) and the aerosol stream entering from the main line (flow rate $Q_{A}$ ). The dilution ratio, $R$, in each branch is given by the correlation:

$$
R=\frac{Q_{A}}{Q_{A}+Q_{D}}
$$

or, since $Q_{T}+Q_{S}=Q_{D}+Q_{A}$ (with the assumption of uniform pressure and temperature in the system).

$$
R=\frac{Q_{T}+Q_{S}-Q_{D}}{Q_{T}+Q_{S}}
$$

The flow rates $Q_{T}, Q_{S}$, and $Q_{D}$ are controlled by the action of critical orifices; consequently the dilution ratios are fixed solely to flow through the critical orifices. 


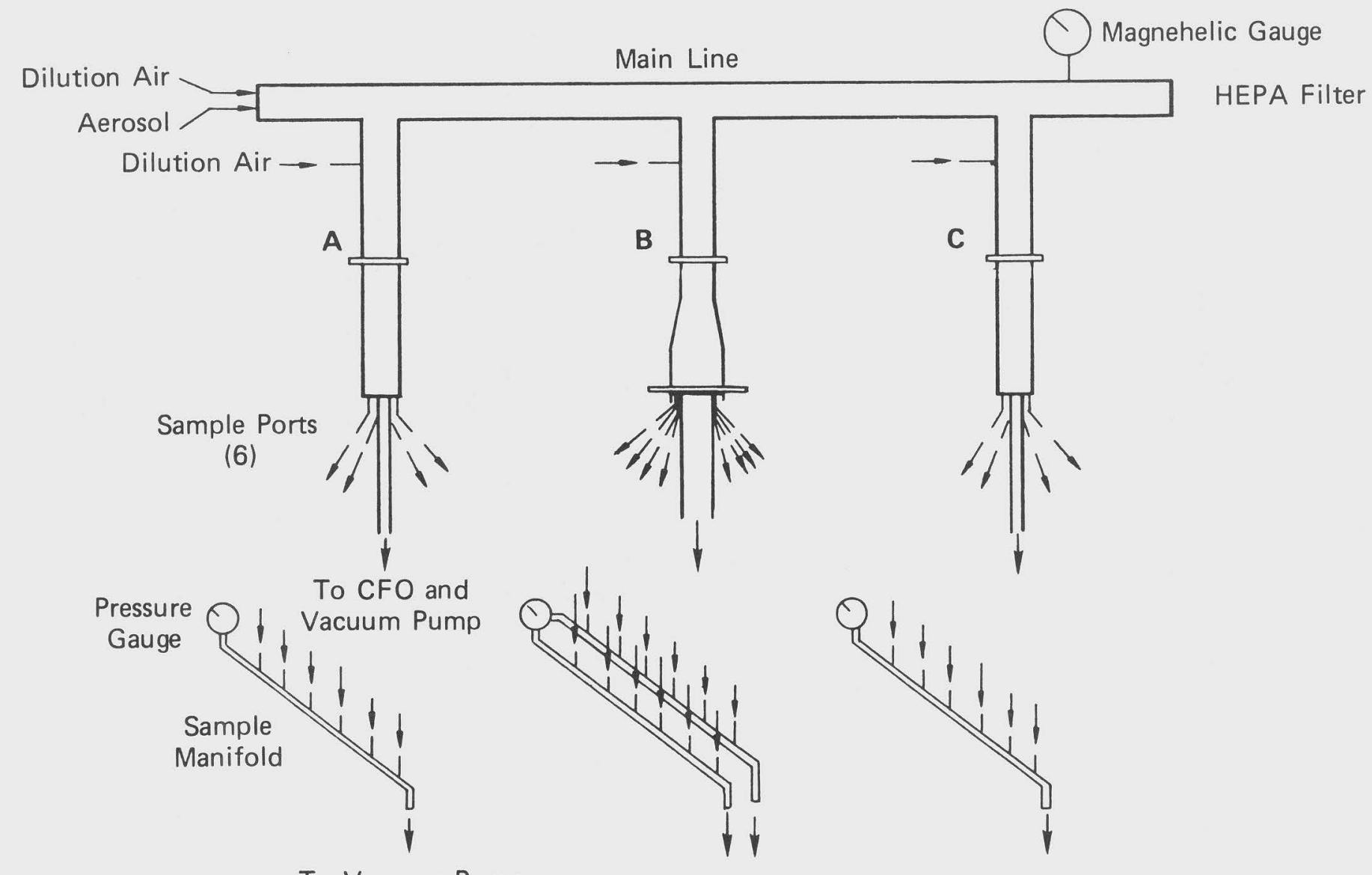

FIGURE 5 ADL AEROSOL DILUTION AND SAMPLING SYSTEM 


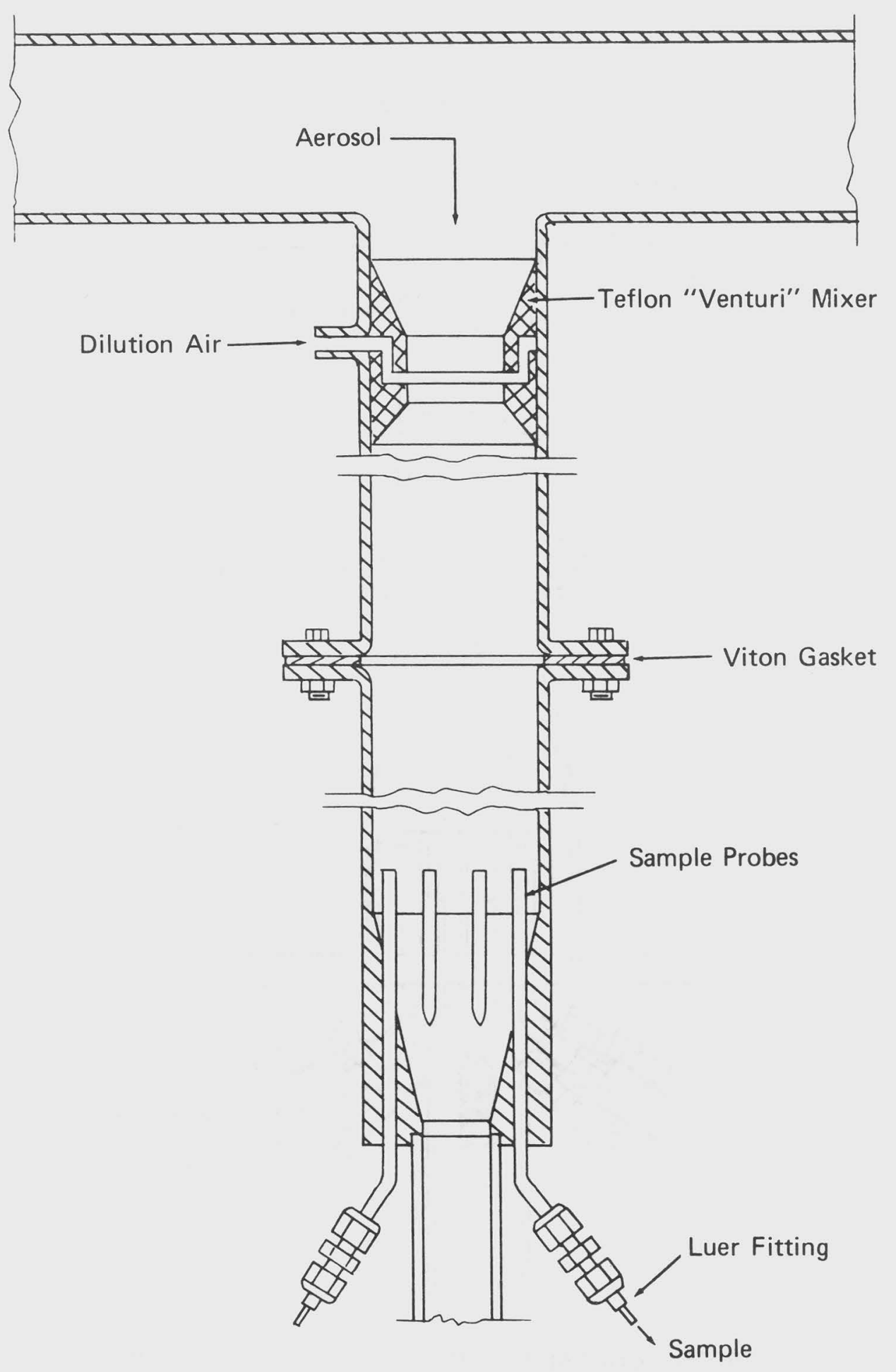

FIGURE 6 CROSS-SECTIONAL VIEW OF DILUTION AND SAMPLING SECTION (Branches $\mathrm{A}$ and $\mathrm{C}$ ) 


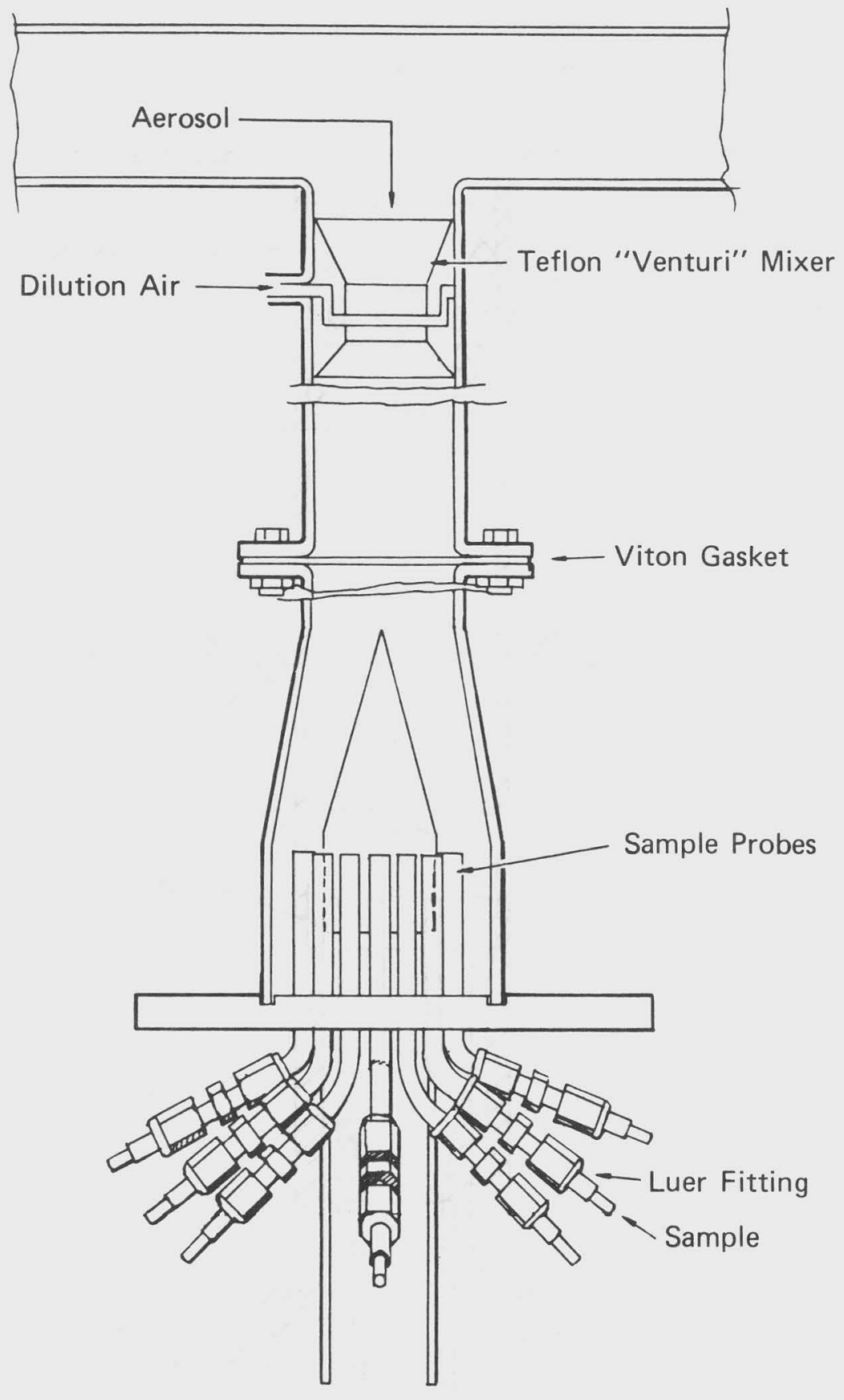

FIGURE 7 CROSS-SECTIONAL VIEW OF DILUTION AND SAMPLING SECTION (Branch B) 
Air Supply--Air from the house compressed air system is treated by successive passage through a cotton filter, a silica gel bed, and a high efficiency glass fiber filter, and a membrane filter. These collections remove, respectively, oil and water droplets, water vapor, and fine particles.

The treated air then passes to two parallel air supply manifolds, each of which is equipped with valves for controlled air flow to various parts of the generation/dilution system. One of the manifolds supplies air to the dilution system. The second supplies air to the aerosol generator, either directly or through calibrated rotameters as may be required by the particular generator being used. Pressure in each manifold is maintained at a fixed level by Moore Nullmatic Pressure regulators and is measured with bourdon gauges (6-inch Ashcroft test gauges). 


\section{REPORTING}

\section{ROUTINE PROJECT COMMUNICATIONS}

The progress in methods development and validation efforts was reported to the NIOSH project officer (PO) on a weekly basis via telephone calls from SRI and ADL. Weekly telephone communications were also made between SRI and ADL. These communications provided a very useful and timely exchange of information. Written progress reports were submitted to the PO monthly.

Quarterly meetings were held by the project steering committee to discuss progress, problems, and plans for future work. The committee was composed of project management staff from NIOSH, SRI, and ADL. These meetings were held at locations alternating between the offices of NIOSH, SRI, and ADL, in addition to laboratories (Los Alamos Scientific Laboratory and OSHA) where information could be gained that would benefit the project effort. Meetings were also held in conjunction with technical society meetings.

\section{METHOD REPORTS}

Upon successful completion of validation studies for a particular substance, the following three draft reports were prepared in a specified format and submitted to NIOSH for review:

- Sampling and Analytical Method (SAM)--This report describes the method of collection of the sample and subsequent analysis. Data regarding the useful range, interferences, precision, and accuracy of the method are summarized.

- Sampling Data Sheet (SDS)--This is a guide for the person collecting samples in the field. Detailed instructions are given for sample collection and shipment of samples to the testing laboratory.

- Backup Data Report (BUD)--This document describes the procedures used to validate the method and includes supporting experimental data, a description of the test atmosphere generation system and the method used to determine atmospheric concentration, a discussion of any problems with the method, and an evaluation of the precision and accuracy.

The methods validated in this program are classified by NIOSH as Class B methods with "accepted" status. A summary of the validated methods appears in Appendix A, Table A-1.

If a valid method did not result from the validation effort, a failure report was submitted, which discussed the experimental approach, reasons for failure, 
and recommendations for further work. The philosophy of this report was to provide a thorough background in the chemistry of the particular substance with regard to sampling and analytical methodology which could be a useful guide or starting point for future work. A list of these reports is in Appendix A, Table A-2.

On occasion, a method was not validated because a single criterion was not met-for example, storage stability of samples was questionable at room temperature, accuracy was not thoroughly evaluated, or further testing of a newly developed sorbent was recommended. In this case, if the method had the potential for providing precise and accurate results with the aid of correction factors or special sample treatment, the method was submitted as a Class E method with "proposed" status. A SAM, SDS, and BUD were prepared as above for a validated method. In addition, a failure report cover page discussed the reasons why the method was not validated, its limitations, and what testing was needed to upgrade the method. A summary of these methods also appears in Appendix A, Table A-1.

\title{
DELIVERY AND REVIEW OF DRAFT REPORTS
}

Several months before the report was received at NIOSH, a technical reviewer was assigned from the Inorganic Methods Development Section or the Organic Methods Development Section of the Measurements Research Branch. In addition to the contract project officer (PO) and the alternate project officer (APO), the reviewer was available to provide technical guidance to SRI or ADL during experimental studies on a particular substance.

In addition to the PO, draft copies of each substance report were submitted for review to:

\author{
Chief of the Industrial Hygiene Section \\ Industry Wide Studies Branch \\ Division of Surveilance \\ Hazard Evaluation and Field Studies
}

and to:

Director of the OSHA Laboratory

Salt Lake City, Utah.

The PO coordinated the review of the substance reports. Two to three weeks after receipt of the reports, the PO and APO met to review the reports and written comments provided by the technical reviewer, the Industrial Hygiene Section, and oSHA. The review covered topics such as determining if the criteria for a validated method were satisfied, safety of the method, contractual requirements, and technical issues. In those cases where the draft report was for a validated method, the report was usually returned with a few minor comments. Sometimes it was necessary to recommend additional work. In rare cases, the data did not support a validated method. However, if the method was sufficiently encouraging, the PO and APO would recommend that a failure report, combined with a Class E method, be written. Otherwise, a failure report was recommended. 
The review of a failure report provided an opportunity to take several approaches:

- Accept the report

- Accept the report, make suggestions for future work and encourage sufficient detail in the report that it was clear why a method could not be developed or validated

- Accept the report and using the data already generated, write a Class E method.

The third approach is not one to be criticized since many times a failure report contained more data than many of the existing Class E methods.

The draft reports were returned with comments and recommendations to SRI or ADL for incorporating into the final reports.

\section{DISTRIBUTION OF METHODS}

Upon receipt of the final methods at NIOSH, the SAMs, SDSs, and BUDs were distributed as follows:

- Coordinator, NIOSH Manual of Analytical Methods

- Coordinator, NIOSH Manual of Sampling Data Sheets

- Chief, Measurements Support Branch, NIOSH

- Chief, Priorities and Research Analysis Branch, NIOSH

- Clearinghouse for Occupational Safety and Health (NIOSH TIC and NTIS)

- OSHA

This distribution allowed for a continuous dissemination (approximately every 3 months) of methods throughout the 3-1/2 year contract. Annual dissemination was accomplished by publishing the methods (SAMs) in the NIOSH Manual of Analytical Methods, Second Edition. The first three volumes of the methods manual were published in April 1977; Volume 4, in August 1978; and Volume 5, in August 1979.

Al1 substances in this study were assigned "compound numbers" in 1974 at the start of the Standards Completion Program, each preceded by the letter "S". The validated methods (Class B) are published in the NIOSH Manual of Analytical Methods in order of their corresponding S-number. The proposed methods (Class E) were each assigned a P\&CAM-number and published in the Manual and ordered by their P\&CAM-numbers. Failure reports were identified with the assigned S-number.

The SDS reports have a similar chronology as the SAMs in the Methods Manual and are published in the NIOSH Manual of Sampling Data Sheets. The BUD Reports and Failure Reports are compiled and published as separate documents.

A11 publications are available through the National Technical Information Service. 


\section{CONCLUSIONS AND RECOMMENDATIONS}

The completion of this research effort represents a significant accomplishment by NIOSH, and its contractors, SRI International and Arthur D. Little, Inc. The Standards Completion Program (SCP) was the first documented attempt by NIOSH to develop and validate a large number of methods against a set of experimental and statistical criteria. As an extension of the SCP, this study resulted in a considerable addition to industrial hygiene sampling and analytical methodology. Of the 130 substances studied on this program, methods were developed and validated for 92 substances. An additional nine methods were deemed useful and may be validated with further testing. These methods will provide a means by which worker exposure to specific toxic substances can be determined. The methods have proven to be precise and accurate under rigorous laboratory testing. Sample collection media have demonstrated more than adequate collection efficiency and capacity for the particular substances. Using these methods, collected samples can be expected to remain stable for at least one week before analysis.

In carrying out this research, we gained a considerable amount of knowledge in the area of sample collection media, including solid sorbents and filters. This knowledge was applied to the development of collection media that were more efficient and convenient to use than bubblers and impingers containing liquid absorbing solutions. New methods of sample treatment and analysis were also investigated and incorporated into methods to achieve greater selectivity and sensitivity: In addition, extensive experience was gained in the generation of test atmospheres of toxic substances at low concentrations. This has enabled us to realistically test sampling and analytical methodology under conditions simulating the industrial environment. Considering the limitations in the scope of work and on the level of effort which could be expended in developmental work, much was accomplished in this program.

Notable specific accomplishments include the development of methods utilizing instrumentation never or rarely used before in NIOSH methods. Without this instrumentation, validated methods may not have been feasible. For example:

- Plasma emission spectroscopy for hafnium, silver, and tantalum

- Ion chromatography for ammonium sulfamate, formic acid, methylamine, and sodium fluoroacetate

- Gas chromatography with photoionization detection for tetraethy1 lead and tetramethyl lead.

- High performance liquid chromatography used more extensively, 20 methods developed

- Polarography for formaldehyde and crotonaldehyde 
Techniques in sample collection and handling to be noted include:

- Active sorbent methods--acid coated silica gel for ammonia and some amines, maleic anhydride coated Chromosorb 104 for cyclopentadiene, silver-coated Chromosorb P for mercury, triethanolamine-coated molecular sieve for nitrogen dioxide, mercuric cyanide-coated silica gel for phosphine, and mercuric chloride-coated silica gel for stibine.

- Girard T reagent for collection of aldehydes.

- Solid sorbents used extensively, 49 methods developed

- Thermal desorption apparatus designed and fabricated in methods for 2-aminopyridine and $\alpha$-chloroacetophenone.

Some discussion is in order regarding the usage of the methods by other laboratories, how it relates to certain aspects of the experimental protocol used in this study, and possible deficiencies that may arise in special situations. It should be emphasized that this does not pertain to a majority of the methods, but rather points out some areas of which users should be aware.

First, this study has been entirely a laboratory validation effort and the effects of potential interfering materials that one may encounter under field conditions have not been thoroughly studied. Within the scope of the project, known interferences were evaluated, but they could not be tested in detail. Obvious samples of situations that may be encountered are, for instance, deactivation of sorbents by co-pollutants or reaction of the analyte with other materials on the collection medium. Considerable modification of GC conditions may be required for the analysis of field samples, especially when determination is attempted for a very toxic substance in the presence of relatively high concentrations of several other compounds.

The methods were tested over an atmospheric concentration range of approximately 0.5 to 2 times the present OSHA environmental limit. To extend the range lower or higher, one must proceed carefully and perform tests accordingly. Reduced analytical recoveries, limited detection capabilities of analytical instrumentation, and poor precision are major obstacles to be considered when trying to extend the range lower. Analytical recoveries of 1 ess than $90 \%$ should not be considered acceptable for most substances, since complications could result in determination of the actual field sample concentration. Breakthrough capacity of a sorbent is a major factor when extending the upper limits of the range.

The methods used for determining desorption efficiency (DE) or analytical method recovery (AMR) have often been criticized as inappropriate, since the application of an analyte in solution does not simulate collection from air. In those cases where an accurate independent determination of the test airborne concentration was made during the validation study, the use of an incorrect DE or AMR would have resulted in poor agreement of the "taken" and "found" concentrations, and a remedy sought. When this comparison was not possible, an inappropriate recovery may have occurred. Since users of the method must generally rely on the DE or AMR determined by their own spiking experiments, we decided in early studies 
that, when possible, a combination of collection medium and solvent would be used that yielded at 1east a $90 \%$ analytical recovery. Thus, an increase in recovery caused by other compounds present in field samples would not cause an appreciable error.

A significant accomplishment in this program included the initiation of testing and validation criteria for high vapor pressure particulates. The need for sampling particulate/vapor mixtures of single substances arose frequently. The criteria developed for evaluating the filter/sorbent methods included the testing of each part of the sampling device over an extended concentration range, and analyzing in detail the results of the breakthrough test and storage stability test. All possible concentration ratios can not feasibly be tested and it may be more appropriate in further investigations to test a "worst case" situation. This may be accomplished by performing breakthrough tests at elevated temperatures. One of the problems that may occur with a sample collected on a filter/sorbent sampling train is that a significant amount of vapor may be absorbed by the filter. In most methods, the filter was combined with the sorbent in a glass vial immediately after collection to prevent loss of vapor from the filter when stored. The combined sample was then analyzed. This procedure calls for complete or identical recoveries from both the filter and the sorbent. Thus, evaluation of analytical recoveries and selection of an efficient filter/sorbent combination is a critical step in the validation effort. The criteria for testing should also address the manner in which analytical recoveries are determined. Satisfactory recovery should be demonstrated separatley for each sampling medium and possibly for the combined media. The development of a sampling device that contains the filter and sorbent combined in either the cassette or tube would be an improvement over the two-part sampling train.

A11 methods developed and validated on this program are not intended to be the ultimate methods of sampling and analysis for toxic substances found in the workplace. Even though the methods were rigorously tested under the validation efforts in this program, certain modifications may be desirable to make a method more useful. In addition, as new sampling and analytical methodology is developed, it may be advantageous to incorporate it into existing methods or replace old methods entirely. It should be emphasized that when a method is modified it must be revalidated to assure precise and accurate results. A number of suggestions are summarized below as a guide for future work in the development of new methods and in improvement of existing methods.

Based upon results obtained from experimental work on substances for which no method was developed, further work is needed for certain compound classes. A11 the halogens and certain reactive halogenated species need to be studied in a concentrated effort. Because of the reactivity of halogens, any collection method for these substances should involve in situ derivatization, preferably with a coated sorbent. The analytical method would have to be specific to distinguish between the individual halogens and other interfering substances. A general method for the halogens would be useful, but may be an impossibility. It would definitely be a challenging future objective. Validated methods are also needed for reactive substances such as methyl and ethyl mercaptan, the isocyanates, and some amines. These substances also usually require derivatization to stabilize the collected sample before analysis. 
There is a definite need for improvement in methods that require cumbersome sampling equipment such as gas sampling bags, bubblers, or impingers containing liquid absorbing solutions. Passive or active sorbents should be developed and tested to replace liquid absorbing reagents. A reliable integrated gas sampler should be developed to replace the present bag sampling technique. The methods should be changed or upgraded with the arrival of new innovative sampling methodology. For example, many methods based upon sorbent tube collection may be adapted to the passive monitor or badge concept of sampling. In the latter case, the sorbent is contained in a badge that is attached to the worker near the breathing zone. The sample is collected by diffusion through a membrane to the sorbent, thus eliminating the personal sampling pump. Of course, this concept must be thoroughly investigated and results demonstrated to be equivalent to those obtained from conventional personal sampling methods.

The source of storage stability problems or poor recoveries from some sorbents could not be easily explained in some cases. These occurrences reflect the need for further study of the composition of the sorbents, the effectiveness of cleaning procedures, and the chemical effect of impurities.

An effort was made in this program to develop methods that could be applied to classes of compounds, rather than single substances. Further work in this area would result in more practical and useful methods. This would also include developing methods that are suitable for a particular industry. For example, if workers were being exposed to a number of solvents in a single industry, the sampling and analytical method should be capable of determining all the solvents.

A very important resource in determining the usefulness of a newly developed method is feedback from users. This information is often not voiced or received so that others may benefit from it. In the course of upgrading present methods or developing new methods, input from users would be very valuable. The NIOSH Manual of Analytical Methods would be an appropriate place to 1 ist the pertinent NIOSH office, address, phone number, and contacts, where the industrial hygienst or chemist can obtain information or give suggestions. A number of self-addressed postcards attached to the manual may encourage more feedback.

The information contained in this report is the culmination of $3-1 / 2$ years of intensive work. The basic goal of this effort was to develop and validate methods which ultimately benefit workers exposed to toxic substances. Although time and funds have limited the exploration of many aspects of sampling and analysis, the objectives of this study have been met. But much work remains to be performed in the development and continual updating of methods, development of new instrumentation, extending the limits of detection, and investigation of all aspects of worker protection. 


\section{APPENDIX A}

This appendix lists all methods validated in this program and gives pertinent information such as the collection medium, sample treatment procedure, analytical method, and the range over which the method was validated (see Table A-1). The code designates whether SRI (S) or ADL (A) performed the experimental work on the substance. The compound numbers were assigned to each substance at the beginning of the Standards Completion Program, and the methods are arranged according to their corresponding S-number in the NIOSH Manual of Analytical Methods.

In addition, proposed (Class E) methods are summarized, each with a corresponding P\&CAM-number that was assigned to it before publication in the Manual. A list of the substances that failed in the validation effort is also included (see Table A-2). 
Table A-1 Validated Methods

\begin{tabular}{|c|c|c|c|c|c|c|c|}
\hline Code & $\begin{array}{l}\text { Compound } \\
\text { No. }\end{array}$ & Analyte & $\begin{array}{l}\text { OSHA standard } \\
(\mathrm{mg} / \mathrm{Cu} \mathrm{m})\end{array}$ & Collection medium & Sample treatment * & $\begin{array}{l}\text { Analytical } \\
\text { method }\end{array}$ & $\begin{array}{l}\text { Range } \\
(\mathrm{mg} / \mathrm{cu} \mathrm{m})\end{array}$ \\
\hline $\mathbf{s}$ & $\$ 345$ & Acetaldehyde & 360 & Bubbler (Girard T) & - & HPLC & $170-670$ \\
\hline s & $\$ 169$ & Acetic ac1d & 25 & Charcoal & Formic ac1d & GC/FID & $12.5-50$ \\
\hline $\mathbf{s}$ & S342 & Alkyl mercury compounds & $\begin{array}{l}0.01 \text { (TWA) } \\
0.04 \text { (C) }\end{array}$ & Carbosleve B & Thermal desorption & $\begin{array}{l}\text { Flame- } \\
\text { less AA }\end{array}$ & $\begin{array}{ll}.004-.017 & \text { (TWA) } \\
.02-.08 \quad \text { (C) }\end{array}$ \\
\hline $\mathbf{A}$ & S346 & Allyl glycidyl ether & 45 & Tenax GC & D1ethyl ether & GC/FID & $19-87$ \\
\hline s & S158 & 2-Aminopyridine & 2 & Tenax GC & Thermal desorption & GC/FID & $0.91-3.60$ \\
\hline S & 5347 & Anmonia & 35 & $\begin{array}{l}\mathrm{H}_{2} \mathrm{SO}_{4} \text { treaced } \\
8 \mathrm{Hlica}_{\mathrm{Cel}}\end{array}$ & $0.1 \mathrm{~N} \mathrm{H}_{2} \mathrm{SO}_{4}$ & ISE & $17-68$ \\
\hline$A$ & s348 & Ammonium sulfamate & 15 & MCEF & Water & IC/ECond & $6.4-27.3$ \\
\hline s & $\$ 163$ & Anlsidine (ortho \& para isomers) & 0.5 & $X A D-2$ & Methanol & IIPLC & $0.25-1.16$ \\
\hline $\mathbf{A}$ & S2 & Antimony \& compounds & 0.5 & MCEF & $\mathrm{HCl}$ & AA & $0.258-1.08$ \\
\hline $\mathbf{s}$ & S276 & ANTU & 0.3 & PTFE filter & Methanol & HPLC & $0.128-0.76$ \\
\hline $\mathbf{s}$ & s253 & Benzoyl peroxide & 5 & MCEF & Diethyl ether & HPLC & $3.12-19.10$ \\
\hline $\mathbf{A}$ & $\$ 138$ & n-Butylamine & $15(\mathrm{C})$ & $\begin{array}{l}\mathrm{H}_{2} \mathrm{SO}_{4} \text { ireated } \\
8111 \mathrm{ca} \text { gel }\end{array}$ & $50 \%$ aq. methanol & GC/FID & $8.1-35.5$ \\
\hline s & \$350 & n-Butyl mercaptan & 35 & Chromosorb 104 & Acetone & GC/FPD-S & $16.8-74$ \\
\hline A & \$313 & Cadmium fume & $\begin{array}{l}0.1 \text { (TWA) } \\
0.3 \text { (C) }\end{array}$ & MCEF & $\mathrm{HNO}_{3} / \mathrm{HCl}$ & $\mathbf{A A}$ & $\begin{array}{l}0.04-0.175 \text { (TWA) } \\
0.122-0.57 \text { (C) }\end{array}$ \\
\hline $\mathbf{A}$ & 5249 & Carbon dioxide & $5000 \mathrm{ppm}$ & Bag & - & GC/TCD & 2270-10, 09p \\
\hline $\mathbf{A}$ & S340 & Carbon monoxide & $50 \mathrm{ppm}$ & Bag & - & $\begin{array}{l}\text { Electro- } \\
\text { chemical }\end{array}$ & $\begin{array}{c}24.7-115.4 \\
\mathrm{Ppm}\end{array}$ \\
\hline A & S278 & Chlordane & 0.5 & $\begin{array}{l}\text { MCEF/Ch romosorb } \\
102\end{array}$ & Toluene & GC/ECD & $0.156-1.17$ \\
\hline S & S11 & Chloroaceraldehyde & $3(\mathrm{C})$ & S111ca gel & $50 \%$ aq. methanol & $\mathrm{GC} / \mathrm{ECD}$ & $1.8-6.4$ \\
\hline s & $\$ 120$ & Chlorodiphenyl, $42 \% \mathrm{Cl}$ & 1 & $\begin{array}{l}\text { Glass fiber filter/ } \\
\text { Bubbler (1sooctane) }\end{array}$ & Isooctane & GC/ECond & $0.51-2.7$ \\
\hline A & $\$ 211$ & 1-Chloro-1-nitropropane & 100 & Chromosorb 108 & Ethyl acetate & GC/FID & $51-206$ \\
\hline $\mathrm{s}$ & $\$ 112$ & Chloroprene & 90 & Charcoal & Carbon d1sulfide & GC/FID & $44-174$ \\
\hline $\mathbf{A}$ & S203 & Cobalt metal fune \& dust & 0.1 & MCEF & Aqua reg1 & $\mathbf{A}$ & $\begin{array}{l}0.031-0.22 \text { (f) } \\
0.040-0.26 \text { (d) }\end{array}$ \\
\hline $\mathbf{s}$ & S354 & Copper fume & 0.1 & MCEF & $\mathrm{HNO}_{3}$ & $\mathbf{A}$ & $0.05-0.37$ \\
\hline $\mathbf{S}$ & \$356 & Crag herbicide & 15 & MCEF & $\begin{array}{l}\text { Water/methylene } \\
\text { blue complex }\end{array}$ & Coloz. & $5-27$ \\
\hline $\mathbf{s}$ & \$279 & $2,4-D$ & 10 & Glass fiber filter & Methanol & HPLC & $5.1-20.3$ \\
\hline $\mathbf{s}$ & $\mathbf{S 2 8 0}$ & Demeton & 0.1 & MCEF/XAD-2 & Toluene & GC/FPD-P & $0.06-0.33$ \\
\hline $\mathbf{s}$ & $\$ 111$ & D1chlorodifluoromethane & 4950 & Charcoal & Methylene chloride & GC/RID & $2940-10,500$ \\
\hline $\mathbf{A}$ & $\$ 109$ & D1chloromonof luoromethane & 4200 & Charcoal & Carbon disulfide & GC/EID & $1730-7600$ \\
\hline $\mathbf{s}$ & $\$ 108$ & Dichlorotetraf luoroethane & 7000 & Charcoal & Merhylene chloride & GC/FID & $3500-14,100$ \\
\hline s & $\$ 140$ & Diechylaninoethanol & 50 & S111ca gel & $\begin{array}{l}\text { Ac1dify with } \mathrm{HCl}, \\
\text { desorb w/MeOH-H } 0 \\
\text { basify w/MaOH-HeOH }\end{array}$ & GC/FID & $25-113$ \\
\hline
\end{tabular}


Table A-1 (continued)

\begin{tabular}{|c|c|c|c|c|c|c|c|}
\hline Code & $\begin{array}{l}\text { Compound } \\
\text { No. }\end{array}$ & Analyte & $\begin{array}{c}\text { OSHA standard } \\
(\mathrm{mg} / \mathrm{cu} \mathrm{m})\end{array}$ & Collection medium & Sample treatment * & $\begin{array}{l}\text { Analytical } \\
\text { method }\end{array}$ & $\begin{array}{l}\text { Range } \\
\text { (mg/cu m) }\end{array}$ \\
\hline $\mathbf{A}$ & \$141 & Dilsopropylamine & 20 & $\begin{array}{l}\text { Impinger/ } \\
0.1 \mathrm{~N} \mathrm{H}_{2} \mathrm{SO}_{4}\end{array}$ & $0.3 \mathrm{~N} \mathrm{KOH}$ & GC/FID & $8.5-37.4$ \\
\hline s & $\mathbf{S 2 1 4}$ & Dinitrobenzene ( 11 isomers) & 1 & $\begin{array}{l}\text { MCEF/Bubbler } \\
\text { (Ethylene glycol) }\end{array}$ & - & HPLC & $0.42-2.4$ \\
\hline $\begin{array}{l}\text { s } \\
\text { s }\end{array}$ & $\$ 166$ & Dinitro-o-cresol & 0.2 & $"$ & - & HPLC & $0.070-0.62$ \\
\hline $\begin{array}{l}\text { s } \\
\text { s }\end{array}$ & $\$ 215$ & Dinitrotoluene & 1.5 & $"$ & - & HPLC & $0.90-5.0$ \\
\hline s & S24 & Diphenyl & 1 & Tenax GC & $\mathrm{CCl}_{4}$ & GC/EID & $0.64-2.4$ \\
\hline $\mathbf{s}$ & $\$ 284$ & Endrin & 0.1 & $\begin{array}{l}\text { MCEF/Chromosorb } \\
102\end{array}$ & Toluene & GC/ECD & $0.06-0.31$ \\
\hline A & $\$ 361$ & 2-Ethoxyethanol & 740 & Charcoal & $\begin{array}{l}\text { Methanol/methylene } \\
\text { chlor1de }\end{array}$ & GC/FID & $340-1460$ \\
\hline $\mathbf{A}$ & $\$ 105$ & Ethyl chloride & 2600 & Charcoal & Carbon disulfide & GC/FID & $1590-6500$ \\
\hline A & S102 & Fluorotrichlorosethane & 5600 & Charcoal & Carbon disulfide & GC/EID & $2390-10,500$ \\
\hline s & S327 & Formaldehyde & $\begin{aligned} 3 \mathrm{ppm} & \text { (TWA) } \\
10 \mathrm{ppm} & \text { (Peak) } \\
5 \mathrm{ppm} & \text { (C) }\end{aligned}$ & Bubbler (Girard T) & - & $\begin{array}{r}\text { Polax. } 1 \\
4\end{array}$ & $\begin{array}{l}1.4-6.2 \text { PPm } \\
\text { (TWA) } \\
4.6-19.8 \mathrm{ppm} \\
\text { (Peak) }\end{array}$ \\
\hline $\mathbf{S}$ & S173 & Formic acid & 9 & Chromosorb 103 & Water & IC/ECond & $4.4-21.6$ \\
\hline s & s17 & Furfural & 20 & Bubbler (Girard T) & - & HPLC & $10.1-40$ \\
\hline s & $\$ 365$ & Furfuryl alcohol & 200 & Porapak Q & Acetone & GC/FID & $120-470$ \\
\hline $\mathbf{A}$ & $\$ 194$ & Hafnium & 0.5 & MCEF & $\begin{array}{l}\mathrm{HNO}_{3} \text {, perchlor } 1 \mathrm{c} \\
\text { ac1d, HF }\end{array}$ & $\begin{array}{l}\text { Plasma } \\
\text { emiseion } \\
\text { epectro. }\end{array}$ & $0.26-1.05$ \\
\hline s & $\$ 287$ & Heptachlor & 0.5 & Chromosorb 102 & Toluene & GC/EC & $0.23-1$ \\
\hline$A$ & $\$ 288$ & Hydrogen cyanide & 11 & $\begin{array}{l}\text { MCEF/Bubbler } \\
(0.1 \mathrm{~N} \mathrm{KOH})\end{array}$ & - & ISE & $5.2-21.0$ \\
\hline $\mathbf{A}$ & S57 & Hydroquinone & 2 & MCEF & Acet1c acid & HPLC & $0.84-4.05$ \\
\hline $\mathbf{s}$ & S366 & Iron oxide fume & 10 & MCEF & $\mathrm{HCl} / \mathrm{HNO}_{3}$ & $\mathbf{A}$ & $3.9-18.2$ \\
\hline $\mathbf{s}$ & s370 & Malathion & 15 & Glass fiber fllter & Isooctane & GC/PPD-P & $8-35$ \\
\hline s & S85 & $\begin{array}{l}\text { MAPP (methyl acetylene/propa- } \\
\text { diene } m(x)\end{array}$ & $1000 \mathrm{ppm}$ & Bag & - & FID & 480-1990 ppm \\
\hline s & $\$ 199$ & Mercury & 0.1 (C) & $\begin{array}{l}\text { Ag coated Chromo- } \\
\text { sorb P }\end{array}$ & Thermal desorption & $\begin{array}{l}\text { Flameless } \\
\text { AA }\end{array}$ & $18 \quad 0.046-0.18$ \\
\hline s & 5371 & Methoxychlor & 15 & Glass fiber filter & Isooctane & GC/ECond & $7.7-31$ \\
\hline $\mathbf{s}$ & 584 & Methyl acetylene & $1000 \mathrm{ppm}$ & Bag & - & GC/RID 52 & $520-1880 \mathrm{ppm}$ \\
\hline s & 5148 & Methylanine & 12 & Silica gel & $\begin{array}{l}\text { Acldify with HCl, } \\
\text { desorb with } \mathrm{H}_{2} \mathrm{O}\end{array}$ & IC/ECond & $6.24-28.1$ \\
\hline $\mathbf{s}$ & s99 & Methyl chloride & $\begin{array}{l}100 \mathrm{ppm} \text { (TWA) } \\
200 \mathrm{ppm} \text { (C) } \\
300 \mathrm{ppm} \text { (Peak) }\end{array}$ & Charcoal & Methylene chloride & $\begin{array}{r}\text { GC/FID } 5 \\
1\end{array}$ & $\begin{array}{l}\text { 59-220 ppm } \\
\text { (TWA) } \\
\text { 143-580 ppm } \\
\text { (Peak) }\end{array}$ \\
\hline $\mathrm{s}$ & 5374 & Methylcyclohexanol & 470 & Charcoal & Methylene chloride & GC/FID & $215-920$ \\
\hline$s$ & S375 & Methylcyclohexanone & 460 & Porapak Q & Acetone & GC/FID & $210-850$ \\
\hline $\mathbf{s}$ & $\$ 291$ & Methyl formate & 250 & Carbosleve B & Ethyl acetate & GC/EID & $108-542$ \\
\hline
\end{tabular}


Table A-1 (continued)

\begin{tabular}{|c|c|c|c|c|c|c|c|}
\hline Code & $\begin{array}{c}\text { Compound } \\
\text { No. } \\
\end{array}$ & Analyte & $\begin{array}{c}\text { OSHA standard } \\
(\mathrm{mig} / \mathrm{cu} \mathrm{m})\end{array}$ & Collection medium & Sample treatment * & * Analytical & $\begin{array}{l}\text { Kange } \\
(\mathrm{mg} / \mathrm{cu} \mathrm{m})\end{array}$ \\
\hline A & $\$ 43$ & Methy1 methacrylate & 410 & $\mathrm{XAD}-2$ & $\mathrm{Cs}_{2}$ & GC/FID & $193-725$ \\
\hline A & S319 & N1tric ac1d & 5 & Impinger (water) & - & ISE & $2.6-10.8$ \\
\hline A & S321 & Nitric oxide & $25 \mathrm{ppm}$ & $\begin{array}{l}\text { Draeger oxidizer, } \\
\text { TEA coated Molecular } \\
\text { Sleve }\end{array}$ & ar & Color. & $11.1-48 \mathrm{ppm}$ \\
\hline A & s7 & p-Nitroaniline & 6 & MCEF & Isopropanol & HPLC & $3.9-12.9$ \\
\hline A & $\mathbf{s} 219$ & N1troethane & 310 & $X A D-2$ & Ethyl acetate & GC/FID & $147-600$ \\
\hline A & s320 & N1trogen dioxide & $5 \mathrm{ppm}$ & $\begin{array}{l}\text { TEA coated Molecuiar } \\
\text { Sleve }\end{array}$ & Ir TEA & Color. & $3.1-11.5 \mathrm{ppm}$ \\
\hline A & $\mathbf{S} 220$ & N1tromethane & 250 & Chromosorb 106 & Ethy1 acetate & GC/AFID & $123-500$ \\
\hline A & $\mathbf{S 2 9 4}$ & Paraquat & 0.5 & PTFE filter & Water & HPLC & $0.256-1.03$ \\
\hline A & $\mathbf{S} 297$ & Pentachlorophenol & 0.5 & MCEF & Ethylene glycol & HPLC & $0.265-1.130$ \\
\hline $\mathbf{A}$ & S296 & Phosdrin & 0.1 & Chromosorb 102 & Toluene & GC/FPD-P & $0.027-0.145$ \\
\hline A & S332 & Phosphine & 0.4 & $\begin{array}{l}\text { HgCN coated sillca } \\
\text { gel }\end{array}$ & Ac1d1c permangana & late Color. & $0.195-0.877$ \\
\hline A & S257 & Phosphorus pentachloride & 1 & $\begin{array}{l}\text { PVC filter/bubbler } \\
\text { (water) }\end{array}$ & $\begin{array}{l}\text { Sodium wolybdate/ } \\
\text { hydrazine sulfate } \\
\text { complexation }\end{array}$ & Color. & $0.55-2.0$ \\
\hline A & S334 & Phosphorus yellow & 0.1 & Tenax GC & Xylene & GC/FPD-P & $0.056-0.244$ \\
\hline A & $S 228$ & Picric acid & 0.1 & MCEF & $70 \%$ aq. methanol & HPLC & $0.036-0.189$ \\
\hline $\mathbf{A}$ & $\mathbf{S} 298$ & Pyrethrum & 5 & Glass fiber filter & Acetonitrile & HPLC & $1.41-8.5$ \\
\hline $\mathbf{A}$ & S181 & Quinone & 0.4 & $\mathrm{XAD}-2$ & Ethanol/hexane & HPLC & $0.17-0.75$ \\
\hline $\mathbf{A}$ & $\mathbf{S 2 9 9}$ & Ronne1 & 10 & $\begin{array}{l}\text { MCEF/Chromosorb } \\
102\end{array}$ & Toluene & GC/FPD-P 2 & $2.82-17.1$ \\
\hline A & S300 & Rotenone & 5 & PTFE filter & Acetonftrile & HPLC & $1.16-11.1$ \\
\hline A & S182 & S1lver, metal and sol. compds. & 0.01 & MCEF & $\mathrm{HNO}_{3}$ & $\begin{array}{l}\text { Plasms } 0.0 \\
18810 n \text { spectro. }\end{array}$ & $.0036-0.0181$ \\
\hline s & S301 & Sod1um fluoroacetate & 0.05 & MCEF & Water & IC/ECond & $0.020-0.137$ \\
\hline A & S381 & Sodium hydroxide & 2 & PTFE f1lter & $\mathrm{HCl}$ & $\begin{array}{l}\mathrm{NaOH} \text { back- } \\
\text { t1tration }\end{array}$ & $0.76-3.9$ \\
\hline A & S243 & Stibine & 0.5 & $\begin{array}{l}\mathrm{HgCl}_{2} \text { coated } \\
\text { silica gel }\end{array}$ & Conc. HCl & $\begin{array}{l}\text { Color. } \\
\text { hodamine B }\end{array}$ & $0.119-1.008$ \\
\hline A & s302 & Strychnine & 0.15 & Glass fiber filter & $\begin{array}{l}\text { HPLC mobile } \\
\text { phase }\end{array}$ & HPLC & $0.073-0.34$ \\
\hline s & S308 & Sulfur dioxide & 13 & Bubbler $\left(\mathrm{H}_{2} \mathrm{O}_{2}\right)$ & $\begin{array}{l}\text { Add isopropyl } \\
\text { alcohol, adjust } \\
\text { pH w/perchloric } \\
\text { acld }\end{array}$ & $\begin{array}{l}\text { Barlum per- } \\
\text { chlorate } \\
\text { titration }\end{array}$ & $6.6-26.8$ \\
\hline S & S244 & Sulfur hexafluoride & 1000 ppm & Bag & - & GC/TCD & 500-2010 ppm \\
\hline s & $\mathbf{S} 245$ & Sulfuryl fluoride & $5 \mathrm{ppm}$ & Bag & - & GC/FPD-S & $\begin{array}{l}2.54- \\
10.29 \mathrm{ppw}\end{array}$ \\
\hline
\end{tabular}

A-4 
Table A-1 (concluded)

\begin{tabular}{|c|c|c|c|c|c|c|c|}
\hline Code & $\begin{array}{c}\text { Compound } \\
\text { No. }\end{array}$ & Analyte & $\begin{array}{c}\text { OSHA standard } \\
(\mathrm{mg} / \mathrm{cu} \mathrm{m})\end{array}$ & Collection medium & Sample treatment * & $\begin{array}{l}\text { Analytical } \\
\text { method }\end{array}$ & $\begin{array}{l}\text { Range } \\
(\mathrm{mg} / \mathrm{cu} \mathrm{m})\end{array}$ \\
\hline $\mathbf{S}$ & \$303 & $2,4,5-T$ & 10 & Glass flber fllter & Methanol & HPLC & $4.9-21.4$ \\
\hline A & S201 & Tantalum & 5 & MCEF & $\begin{array}{l}\mathrm{HNO}_{3}, \text { perchloric } \\
\text { acld and } \mathrm{HF}\end{array}$ & $\begin{array}{l}\text { Plasma } \\
\text { emission } \\
\text { spectro. }\end{array}$ & $2.5-10.0$ \\
\hline A & S383 & Tetraethyl lead & 0.075 & $\mathrm{XAD}-2$ & Pentane & GC/PID & $0.045-0.20$ \\
\hline A & S384 & Tetramethyl 1ead & 0.07 & XAD-2 & Pentane & GC/PID & $0.040-0.18$ \\
\hline s & S256 & Th1ram & 5 & PTFE filter & Acetondtr1le & HPLC & $3.0-12.2$ \\
\hline A & s391 & Vanadium, $\mathrm{V}_{2} \mathrm{O}_{5}$ dust & 0.5 & MCEF & $\mathrm{NaOH}$ & $\mathrm{AA} / \mathrm{HGA}$ & $0.24-0.90$ \\
\hline A & S388 & Vanadium fume & 0.1 (C) & MCEF & $\mathrm{NaOH}$ & $\begin{array}{l}\text { flameless } \\
\text { AA/HGA }\end{array}$ & $0.060-0.29$ \\
\hline A & S316 & Zinc oxlde fume & 5 & PVC filter & - & $\begin{array}{l}\text { X-ray } \\
\text { diffractior }\end{array}$ & $\mathrm{n}^{2.4-9.9}$ \\
\hline
\end{tabular}

\section{Proposed (Class E) Methods}

\begin{tabular}{|c|c|c|}
\hline $\mathbf{A}$ & $\begin{array}{l}\text { P\&CAM } 304 \\
\text { (\$154) }\end{array}$ & $\begin{array}{l}\text { o-Chrorobenzylidene } \\
\text { malononitrile (OCBM) }\end{array}$ \\
\hline s & $\begin{array}{l}\text { P\&CAM } 291 \\
\text { (S9) }\end{array}$ & alpha-Chloroacetophe \\
\hline $\mathbf{s}$ & $\begin{array}{l}\text { P\&CAM } 285 \\
(5177)\end{array}$ & Crotonaldehyde \\
\hline s & $\begin{array}{l}\text { P\&CAM } 294 \\
\text { S83) }\end{array}$ & Cyclopentadiene \\
\hline A & $\begin{array}{l}\text { P\&CAM } 297 \\
(\$ 390)\end{array}$ & Dibutyl phosphate \\
\hline $\mathbf{A}$ & $\begin{array}{l}\text { P\&CAM } 295 \\
(S 282)\end{array}$ & Dichlorvos (DDVP) \\
\hline s & $\begin{array}{l}\text { P\&CAM } 302 \\
(5180)\end{array}$ & Maleic anhydride \\
\hline A & $\begin{array}{l}\text { P\&CAM } 315 \\
\text { (S305) }\end{array}$ & TEPP \\
\hline A & $\begin{array}{l}\text { P\&CAM } 313 \\
(\mathrm{~S} 307)\end{array}$ & Warfarin \\
\hline
\end{tabular}

\begin{tabular}{|c|c|c|c|c|}
\hline 0.4 & $\begin{array}{l}\text { PTFE f } 11 \text { ter/ } \\
\text { Tenax GC }\end{array}$ & $\begin{array}{l}20 \% \text { methylene } \\
\text { chloride in hexane }\end{array}$ & HPLC & $0.147-0.82$ \\
\hline 0.3 & Tenax GC & Thermal desorption & GC/FID & $0.18-0.62$ \\
\hline 6 & $\begin{array}{l}\text { Bubbler } \\
\text { (hydroxylamine) }\end{array}$ & - & Polar. & $2.9-23.4$ \\
\hline 200 & $\begin{array}{l}\text { Chromosorb } 104 \\
\text { coated with } \\
\text { maleic anhydride }\end{array}$ & Ethyl acetate & GC/F ID & $76-380$ \\
\hline 5 & PTFE filter & $\begin{array}{l}\text { Acetonitrile, } \\
\text { trimethylsilyl } \\
\text { reagent }\end{array}$ & $G C / F P D-P$ & $2.3-10.0$ \\
\hline 1 & $X A D-2$ & Toluene & GC/FPD-P & $0.38-1.71$ \\
\hline 1 & Bubbler $\left(\mathrm{H}_{2} \mathrm{O}\right)$ & - & HPLC & $0.50-2.14$ \\
\hline 0.05 & Chromosorb 102 & Toluene & GC/FPD-P & $0.025-0.124$ \\
\hline 0.1 & PTFE filter & Methano1 & HPLC & $0.054-0.24$ \\
\hline
\end{tabular}

Codes for Analytical Methods

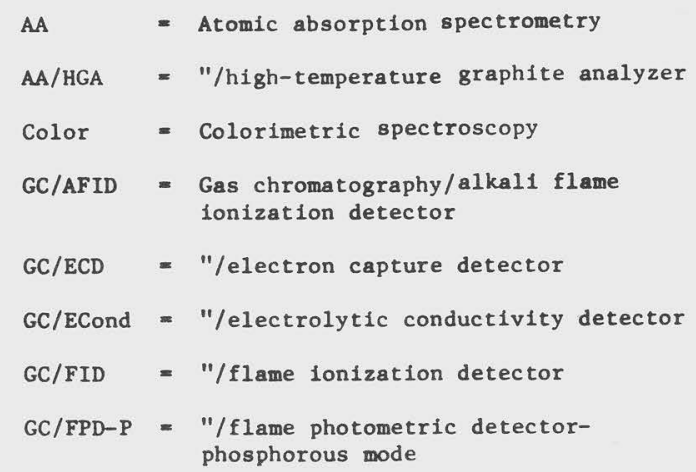

*unless otherwise noted, samples are desorbed/extracted in the solvent indicated. 
Table A-2 Failure Reports

Code

S

s

A

A

A

S

A

A

S

A

A

s

$\mathrm{S}$

S

S

S

S

A

S

A

A

S

A

A
Compound No.

S21

S157

S277

S230

S231

\$322

S212

\$207

S127

S34

S136

S145

S362

S363

S325

S364

S239

S252

S373

S221

S171

S271

S159

S14

S270

S304

S184

S344

S226

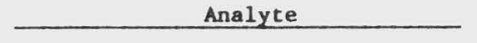

Acrole1n

Acrylamide

Azinphos, methyl

Boron trifluoride

Bromine

Chlorine

Chloropicrin

Dibrom

1,3-Dichloro-5,5-dimethylhydantoin

Dimethylphthalate

Ethanolamine

Ethylenediamine

Ethyl mercaptan

Ferbam

Fluorides in dust

Fluorine

Iodine

Methy1 1socyanate

Methyl mercaptan

1-N1tropropane

Oxaltc actd

Perchloromethyl mercaptan

p-Phenylenediamine

Plval

Sulfur monochloride

TEDP

Tin, organic compounds

2,4-Toluened11socyanate

Trinitrotoluene
OSHA Standard

(mg/cu m)

0.25

0.3

0.1

3 (Ce1ling)

0.7

3

0.7

3

0.2

5

6

25

25

15

2.5

0.2

1

0.05

20

90

1

0.8

0.1

0.1

$1 \mathrm{ppm}$

0.2

0.1

0.14

1.5 


\section{APPENDIX B * \\ STATISTICAL PROTOCOL}

\section{INTRODUCTION}

This appendix gives the steps of a recommended statistical protocol for analysis of air concentration data for hazardous chemicals. The purpose of the statistical analysis is to estimate the total precision error of a subject method and resolve this error into its components due to the sampling method (less pump error), the analytical method (including error in the desorption efficiency factor), and an assumed level of pump error.

\section{MAXIMUM NET ERROR FOR UNBIASED METHOD}

The present suggested standard for air monitoring accuracy is that the absolute total error (sampling plus analysis) should be less than $25 \%$ in at 1 east $95 \%$ of samples analyzed at the level of the standard. In the case of normally distributed sampling and analysis errors (and no bias), the above requirement implies that the true coefficient of variation of the total error, denoted by $\mathrm{CV}_{\mathrm{T}}$, should be no greater than 0.128 derived as follows: $\mathrm{CV}_{\mathrm{T}}=0.25 / 1.96=0.128$. The number 0.128 is the largest true precision for a net error of $\pm 25 \%$ at the $95 \%$ confidence level. The number of 1.96 is the appropriate z-statistic from the standard normal distribution at the same confidence level.

However, the estimated values of $\mathrm{CV}_{\mathrm{T}}$ (denoted by $\overline{\mathrm{CV}}_{\mathrm{T}}$ ), which are reported by SRI under the NIOSH contract 210-76-0123, will themselves be subject to appreciable random errors of estimation. Therefore, the "critical value" not to be exceeded by an experiment 1 value of $\overline{C V}_{\mathrm{T}}$ must be lower than the maximum permissible true value of $\mathrm{CV}_{\mathrm{T}}$ which is implied by the wording of the accuracy standard. The maximum permissible true value of $\mathrm{CV}_{\mathrm{T}}$ will be referred to as its "target level" and the required degree of confidence that a subject method meets this accuracy standard can be obtained by calculating an appropriate $[(1-\alpha)$ probability level] upper confidence limit on $\overline{C V}_{\mathrm{T}}$ and obeying the following decision rule:

Rule: Reject the method if the $95 \%$ upper confidence 1 imit on $\overline{\mathrm{CV}}_{\mathrm{T}}$ exceeds the target level of $\mathrm{CV}_{\mathrm{T}}$. Otherwise, accept the method.

As would be expected, to have the $95 \%$ degress of confidence that $\overline{\mathrm{CV}}_{\mathrm{T}}$ does not exceed its target level, we must also suffer the penalty of sometimes falsely rejecting "good" methods whose true $\mathrm{CV}_{\mathrm{T}}$ is satisfacotry, but for which the $\overline{\mathrm{CV}}_{\mathrm{T}}$

*

Reprinted from Documentation of NIOSH Validation Tests, National Institute for Occupational Safety and Health, Cincinnati, Ohio (DHEW-NIOSH Publication 非7185), 1977. Available from Superintendent of Documents, U.S. Government Printing Office, Washington, DC, Order No. 017-033-00231-2. 
estimate is not precise enough to keep its upper confidence limit below the target value. Such decision errors, referred to as "type-1 errors," will occur randomly but with a controlled frequency of less than $5 \%$ of cases. The $5 \%$ probability of type-1 error is by definition the complement of the confidence level.

As an example, consider the $\overline{\mathrm{CV}}_{\mathrm{T}}$ which is a pooled value determined from three groups of six samples. The $\overline{\mathrm{CV}}_{\mathrm{T}}$ would have to be less than 0.105 in order for us to be $95 \%$ confident that the true $\mathrm{CV}_{\mathrm{T}} \leq 0.128$ (i.e., in order for us to be $95 \%$ confident that future errors by the same method would not exceed $\pm 25 \%$ more than $5 \%$ of the time). The critical value of $\overline{\mathrm{CV}}_{\mathrm{T}}$ is 0.105 at the $\alpha=0.05$ type- 1 error leve1. Thus, for an unbiased method, the largest laboratory-determined $\overline{\mathrm{CV}}_{\mathrm{T}}$ should not exceed 0.105 .

To be reasonably sure that a test method meets the NIOSH accuracy standard, we have adopted the following decision rule: if the estimate $\left(\overline{\mathrm{CV}}_{\mathrm{T}}\right)$ is below 0.105 , the $95 \%$ upper confidence limit on $\mathrm{CV}_{\mathrm{T}}$ would be below $12.8 \%$ and the method would be found satisfactory. This decision rule is based on assumptions that errors are normally distributed and the method is unbiased. Figure B-1 provides adjustments to the critical values of $\overline{\mathrm{CV}}_{\mathrm{T}}$ when a method is biased. The dotted curve gives critical values of $\overline{\mathrm{CV}}_{\mathrm{T}}$ as a function of bias for a statistical significance test performed at the 5\% type-1 error level (i.e., 95\% confidence level).

It is important to note that the critical values of $\overline{\mathrm{CV}}_{\mathrm{T}}$ for Figure B-1 were computed under the assumption that the bias is a known quantity. This was necessary because the protocol contains no standard statistical experimental design for determination of bias by means of an independent method. However, the bias was estimated as the difference between the mean of the test results and the true value (determined from the independent method) divided by the true value. In cases where confidence limits can be calculated for the bias, the critical $\bar{c}_{T}$ should be read from the dotted curve at a position corresponding to the $95 \%$ upper confidence limit for the bias. This is a conservative procedure, but better critical values could only be determined if the standard error of the bias were known.

The caluclated points through which the curves of Figure B-1 were drawn using a French curve are given below:

\begin{tabular}{rrc}
$\begin{array}{c}\text { Bias } \\
(\%)\end{array}$ & $\begin{array}{c}\text { Target } \mathrm{CV}_{\mathrm{T}} \\
(\%)\end{array}$ & $\begin{array}{c}\text { Critical } \overline{\mathrm{CV}}_{\mathrm{T}} \\
(\%)\end{array}$ \\
\cline { 2 - 2 } 0 & 12.8 & 10.5 \\
2.5 & 12.5 & 10.3 \\
5.0 & 11.8 & 9.8 \\
10.0 & 9.1 & 7.9 \\
15.0 & 6.1 & 5.8 \\
16.8 & 5.0 & 5.0 \\
20.0 & 3.0 & (Unattainable) \\
25.0 & 0 & (Unattainable)
\end{tabular}

Since $\mathrm{CV}_{\mathrm{P}}$, the coefficient of variation of pump error, is assumed to be $5 \%$, any method with a bias above $16.8 \%$ could not meet the accuracy standard (no matter 


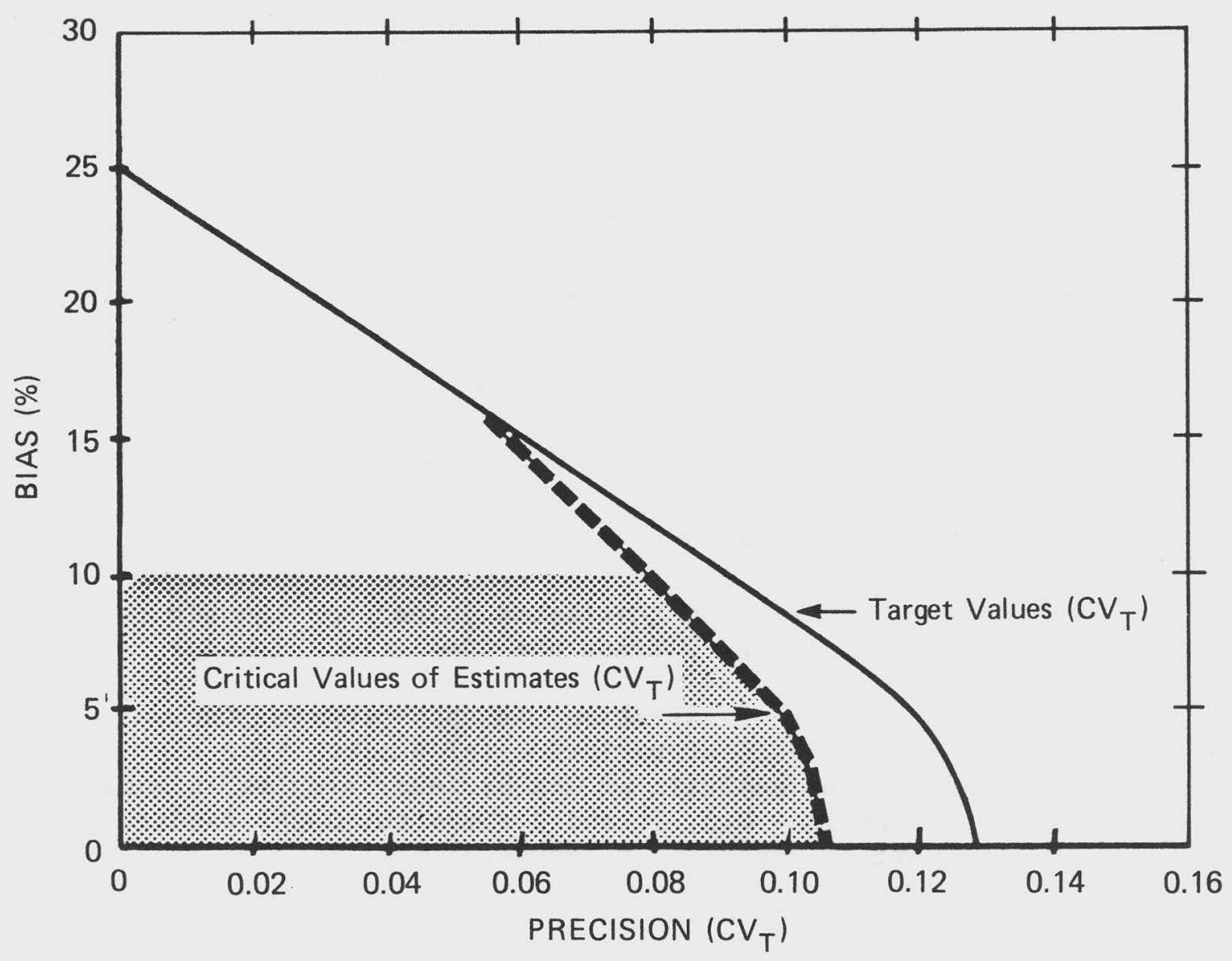

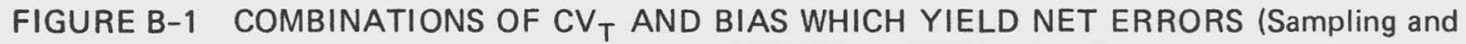
Analysis) OF 25\% OR LESS AT LEAST 95\% OF THE TIME 
how small were the $\mathrm{CV}_{\mathrm{S}}$ and $\mathrm{CV}_{\mathrm{A}}$ for other sampling errors and analysis errors, respectively).

\section{DEFINITIONS AND SYMBOLS}

Mean arithmetic average $(\bar{x})$, defined as the sum of the observations defined by the number of observations ( $n$ ).

Standard deviation

the positive square root of the variance which is defined as the sum of squares of the deviations of the observations from their mean $(\bar{x})$ divided by one less than the number of observations $(n-1)$.

$$
\text { standard deviation }=\sqrt{\frac{\sum_{i=1}^{n}\left(x_{i}-\bar{x}\right)^{2}}{n-1}}
$$

CV coefficient of variation or relative standard deviation, defined as the standard deviation divided by the mean.

$$
\mathrm{CV}=\frac{\text { standard deviation }}{\text { mean }}
$$

$\mathrm{CV}_{1} \quad$ coefficient of variation (estimated value) for the six analytical samples at $0.5,1$, and 2 times the OSHA standard level.

$\mathrm{CV}_{2} \quad$ coefficient of variation (estimated value) for the six generated samples at $0.5,1$, and 2 times the OSHA standard level.

CV pooled coefficient of variation, in this program, the value derived from the coefficients of variation (of a given type e.g., $\mathrm{CV}_{1}$ or $\mathrm{CV}_{2}$ ) obtained from the analysis of six or fewer samples at each of the three test levels - 0.5, 1, and 2 times the OSHA standard level. The mathematical equation is expressed as:

$$
\overline{C V}=\sqrt{\frac{\sum_{i=1}^{3} f_{i}\left(C V_{i}\right)^{2}}{f}}
$$

where:

$\mathrm{f}_{i}=$ degrees of freedom, equal to number of observations $\left(\mathrm{n}_{i}\right)$ minum one, at the $i^{\text {th }}$ level.

$\mathrm{CV}_{i}=$ coefficient of variation $\left(\mathrm{CV}_{1}\right.$ or $\left.\mathrm{CV}_{2}\right)$ of the observations at the $i^{\text {th }}$ concentration level.

$\mathrm{f} \quad=\sum_{i=1}^{3} \mathrm{f}_{\mathrm{i}}$

$i=$ index for the three concentration levels. 
$\overline{\mathrm{CV}}_{1} \quad$ pooled coefficient of variation calculated as above based on data for the 18 analytical (spiked) samples (three groups of six).

$\overline{\mathrm{CV}}_{\mathrm{A}+\mathrm{DE}}$ derived corrected value for $\mathrm{CV}_{1}$ to include precision error due to the use of desorption efficiency factor, which is an average of six values.

$$
\overline{\mathrm{CV}}_{\mathrm{A}+\overline{\mathrm{DE}}}=\overline{\mathrm{CV}}_{1} \sqrt{7 / 6}=1.0801 \overline{\mathrm{CV}}_{1}
$$

$\overline{\mathrm{CV}}_{\mathrm{A}} \overline{\mathrm{ARM}}$ derived corrected value of $\mathrm{CV}_{\mathbf{1}}$ analogous to the desorption efficiency factor noted above except that this notation is used where the factor is associated with analytical method recovery (ARM) other than for solid sorbents.

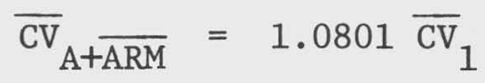

$\overline{\mathrm{CV}}_{2}$ pooled coefficient of variation based on the data for the eighteen generated samples.

$\overline{\mathrm{CV}}_{\mathrm{S}} \quad$ coefficient of variation of the sample collection, not including the variability of the personal sampling pump. The value dependent on the data from the eighteen analytical and eighteen generated samples.

$$
\overline{\mathrm{CV}}_{\mathrm{S}}=\sqrt{\left(\overline{\mathrm{CV}}_{2}\right)^{2}-\left(\overline{\mathrm{CV}}_{1}\right)^{2}} \quad \text { (see "Note" below) }
$$

$\mathrm{CV}_{\mathrm{P}} \quad$ coefficient of variation due to the pump error, assumed to be equal to 0.05 .

Note:

In case $\overline{\mathrm{CV}}_{2}<\overline{\mathrm{CV}}_{1}$, take $\overline{\mathrm{CV}}_{\mathrm{S}}=0$. Then replace $\overline{\mathrm{CV}}_{1}$ by a pooled estimate $\left(\overline{\mathrm{CV}}_{1}{ }^{*}\right)$ based on $\overline{\mathrm{CV}}_{1}$ and $\overline{\mathrm{CV}}_{2}$, namely

$$
\overline{\mathrm{CV}}_{1} * \sqrt{\frac{\mathrm{f}_{1} \overline{\mathrm{CV}}_{1}^{2}+\mathrm{f}_{2} \overline{\mathrm{CV}}_{2}^{2}}{\mathrm{f}_{1}+\mathrm{f}_{2}}}
$$

where $f_{1}$ and $f_{2}$ are the respective $f$-values used in the denominators of $\overline{\mathrm{CV}}_{1}{ }^{2}$ and $\overline{\mathrm{CV}}_{2}{ }^{2}$. 

composite variations in sampling and analysis, desorption efficiency, and pump error.

$$
\overline{\mathrm{CV}}_{\mathrm{T}}=\sqrt{\left(\overline{\mathrm{CV}}_{\mathrm{S}}\right)^{2}+\left(\overline{\mathrm{CV}}_{\mathrm{A}+\overline{\mathrm{DE}}}\right)^{2}+\left(\overline{\mathrm{CV}}_{\mathrm{P}}\right)^{2}}
$$

or:

$$
\overline{\mathrm{CV}}_{\mathrm{T}}=\sqrt{\left(\overline{\mathrm{CV}}_{2}\right)^{2}-\left(\overline{\mathrm{CV}}_{1}\right)^{2}+1.1667\left(\overline{\mathrm{CV}}_{1}\right)^{2}+(0.05)^{2}}
$$

or

$$
\overline{\mathrm{CV}}_{\mathrm{T}}=\sqrt{\left(\overline{\mathrm{CV}}_{2}\right)^{2}+0.1667 \overline{\mathrm{CV}}_{1}{ }^{2}+(0.05)^{2}} \quad \text { (see "Note" page B-5) }
$$

Grubb's test for rejction of an observation is applied in order to determine if one of the observations should be rejected as being an outlier. The following equation was used for the test:

$$
\mathrm{B}_{1}^{\prime}=\frac{\mathrm{x}-\overline{\mathrm{x}}}{\mathrm{s}} \text { or }\left(\frac{\overline{\mathrm{x}}-\mathrm{x}}{\mathrm{s}}\right)
$$

where:

$$
\begin{aligned}
& \mathrm{x}=\text { observation being tested (most distant from the mean) } \\
& \overline{\mathrm{x}}=\text { mean of } \mathrm{n} \text { observations } \\
& \mathrm{s}=\text { standard deviation based on } \mathrm{n}-1 \text { degress of freedom }
\end{aligned}
$$

For any six observations, a value can be rejected if $\mathrm{B}_{1}{ }^{\prime} \geq 1.944$. The $\mathrm{B}_{1}{ }^{\prime}$ limit is based on a $1 \%$ significance level (i.e., a $B_{1}{ }^{\prime}$ value calculated from the data can be expected to exceed 1.944 only $1 \%$ of the time if the observation is a legitimate one conforming to the underlying theory).

Bartlett's test for homogeneity of CVs is applied in order to test the feasibility of "pooling the coefficients of variation" for any set of 18 generated samples (i.e., 6 at each of the $0.5,1$, and 2 times OSHA standard level). The following equation for chi-squared, with two degrees of freedom, was used:

$$
\text { chi-squared }=\frac{f \ln \left(\overline{\mathrm{CV}}_{2}\right)^{2}-\sum_{i=1}^{3} \mathrm{f}_{i} \ln \left(\overline{\mathrm{CV}}_{2 i}\right)^{2}}{1+\frac{1}{3(3-1)}\left[\left(\sum_{i=1}^{3} \frac{1}{\mathrm{f}_{i}}\right)-\frac{1}{\mathrm{f}}\right]}
$$


where:

$$
\begin{aligned}
& \overline{\mathrm{CV}}_{2}=\text { pooled coefficient of variation of } 18 \text { generated samples } \\
& \overline{\mathrm{CV}}_{2 i}=\begin{array}{l}
\text { coefficient of variation of six generated samples at the } \\
\text { th level }
\end{array} \\
& \mathrm{f}_{i}=\begin{array}{l}
\text { degrees of freedom associated with }\left(\overline{\mathrm{CV}}_{2 i}\right)^{2} \text { and equal to } \\
\text { number of observations at the } i^{\text {th }} 1 \text { evel minus one. }
\end{array} \\
& \mathrm{f}=\sum_{i=1}^{3} \mathrm{f}_{i}
\end{aligned}
$$

To pass Bartlett's test at the $1 \%$ significance leve1, chi-squared must be less than or equal to 9.21 (chi-squared has two degrees of freedom). 
